\title{
STRONG RENEWAL THEOREMS WITH INFINITE MEAN
}

\author{
BY \\ K. BRUCE ERICKSON( $\left.{ }^{1}\right)$
}

\begin{abstract}
Let $F$ be a nonarithmetic probability distribution on $(0, \infty)$ and suppose $1-F(t)$ is regularly varying at $\infty$ with exponent $\alpha, 0<\alpha \leqq 1$. Let $U(t)=\sum F^{n^{*}}(t)$ be the renewal function. In this paper we first derive various asymptotic expressions for the quantity $U(t+h)-U(t)$ as $t \rightarrow \infty, h>0$ fixed. Next we derive asymptotic relations for the convolution $U^{*} z(t), t \rightarrow \infty$, for a large class of integrable functions $z$. All of these asymptotic relations are expressed in terms of the truncated mean function $m(t)=\int_{0}^{t}[1-F(x)] d x, t$ large, and appear as the natural extension of the classical strong renewal theorem for distributions with finite mean. Finally in the last sections of the paper we apply the special case $\alpha=1$ to derive some limit theorems for the distributions of certain waiting times associated with a renewal process.
\end{abstract}

1. Principal theorems. Let $F$ be a probability measure concentrated on $[0, \infty)\left({ }^{2}\right)$ and let $U$ be the associated renewal measure defined for any measurable set $I$ by

$$
U\{I\}=\sum_{0}^{\infty} F^{n^{*}}\{I\}
$$

where $F^{n^{*}}$ denotes the $n$-fold convolution of $F$ with itself $\left(F^{0^{*}}\right.$ is the probability measure concentrated at the origin). The series (1.1) converges to a finite number for every bounded $I$. (For this and other elementary properties of $U$ see [3, VI. 6]; for a probabilistic interpretation of $U$ see $\S 9$ in this paper.) We write $U(x)$ for $U\{[0, x]\}$ and we shall henceforth ignore the distinction between $U$ the measure and $U$ the function. (This convention applies to other measures as well.)

The main results of this paper deal primarily with the differences $U(t+h)-U(t)$ for $h>0$ fixed, and $t \rightarrow \infty$. The principal assumption is that $F$ has the form

$$
1-F(t)=t^{-\alpha} L(t), \quad t>0,
$$

Received by the editors October 4, 1969.

AMS Subject Classifications. Primary 6070, 6020, 6030; Secondary 4042, 4252.

Key Words and Phrases. Probability distributions, renewal function, nonarithmetic, regular and slow variation, strong renewal theorem, infinite mean, convolution, waiting times, renewal process, characteristic function, inversion formulas, weak convergence of measures, domain of attraction, local limit theorems, Karamata Tauberian theorem.

(1) The work was supported in part by the National Institute of Health and by a grant from the Graduate School of the University of Wisconsin. I am greatly indebted to Professor Peter Ney for the many valuable discussions regarding this paper. Most of this paper is part of the author's Ph.D. thesis at the University of Wisconsin.

$\left(^{2}\right)$ We assume, however, that not all the mass is at the origin.

Copyright (C) 1970, American Mathematical Society 
where $0 \leqq \alpha \leqq 1$ (fixed) and $L$ is a slowly varying function $\left({ }^{3}\right)$. Unless otherwise indicated, we also assume $F$ is nonarithmetic; that is, we exclude the possibility that $F$ concentrates the entire mass on the multiples of some positive real number. For $\alpha \neq 1$, the arithmetic versions of Theorems 1 and 2 below were treated by $A$. Garsia and J. Lamperti, [5] (nothing was known in the case $\alpha=1$ ). See §2(ii) for further discussion. Define the "truncated mean" function

$$
m(t)=\int_{0}^{t}(1-F(x)) d x=t(1-F(t))+\int_{0}^{t} x F\{d x\} .
$$

THEOREM 1. Let $F$ satisfy (1.2) with $\frac{1}{2}<\alpha \leqq 1$. Then for every $h>0$ and as $t \rightarrow \infty$

$$
U(t+h)-U(t) \sim C_{\alpha} h / m(t)
$$

where $C_{\alpha}=[\Gamma(\alpha) \Gamma(2-\alpha)]^{-1}$.

THEOREM 2. If $0<\alpha \leqq \frac{1}{2}$ then

$$
\liminf _{t \rightarrow \infty} m(t)(U(t+h)-U(t))=C_{\alpha} h .
$$

REMARK. When $\alpha \neq 1, m(t) \sim(1-\alpha)^{-1} t^{1-\alpha} L(t), t \rightarrow \infty$ (see Lemma $1, \S 3$ ) and $\Gamma(\alpha) \Gamma(2-\alpha)=\pi(1-\alpha) \csc \pi \alpha$. It follows that (1.4) is equivalent to

$$
\lim _{t \rightarrow \infty} t^{1-\alpha} L(t)(U(t+h)-U(t))=\frac{\sin \pi \alpha}{\pi} h .
$$

The results of Theorems 2, 3, and 4 may be restated in an analogous fashion.

Let $z$ be a nonnegative function on $[0, \infty)$. For $h>0$ write

$$
\sigma^{-}=h \sum_{k=1}^{\infty} \sup \{z(x):(k-1) h \leqq x<k h\}
$$

and similarly define $\sigma_{-}$with inf in place of sup. Following Feller [3, p. 348], we say that $z$ is directly Riemann integrable (dri) if the series defining the upper sum $\sigma^{-}$converges and $\sigma^{-}-\sigma_{-} \rightarrow 0$ as $h \rightarrow 0$. It follows immediately that a dri function is bounded, measurable and (Lebesgue) integrable.

THEOREM 3. Let $z$ be a nonnegative dri function on $[0, \infty)$ which satisfies

$$
z(t)=O(1 / t), \quad t>0 .
$$

If $F$ has the form (1.2) with $\frac{1}{2}<\alpha \leqq 1$ then

$$
\int_{0}^{t} z(t-y) U\{d y\} \sim \frac{C_{\alpha}}{m(t)} \int_{0}^{\infty} z(x) d x .
$$

$\left({ }^{3}\right)$ A measurable ultimately positive function $L$ on $[0, \infty)$ is regularly varying with exponent $\rho$ if as $t \rightarrow \infty, L(x t) / L(t) \rightarrow x^{\rho}$ for all $x>0$. When $\rho=0$, i.e., $L(x t) / L(t) \rightarrow 1$, we also say $L$ is slowly varying. We assume as known the various properties of slowly varying functions as described in [3, pp. 272-274], or in [6]. Note that the function $L$ in (1.2) must be bounded on bounded subintervals of $[0, \infty)$. 
THEOREM 4. Let $z \geqq 0$ be a dri function (not necessarily satisfying (1.7)). If $F$ satisfies (1.2) with $\alpha \neq 0$ then

$$
\liminf _{t \rightarrow \infty} m(t) \int_{0}^{t} z(t-y) U\{d y\}=C_{\alpha} \int_{0}^{\infty} z(x) d x .
$$

Remarks. 1. Define a complex valued $z$ to be dri if $|z|$ is dri as defined above. With this definition it follows readily from Theorem 3 that (1.8) holds for any dri $z$ satisfying (1.7).

2. Any piecewise continuous function on $[0, \infty)$ vanishing off a compact interval is dri and certainly satisfies (1.7). In particular, taking $z(x)=1$ for $0 \leqq x \leqq h$, and $z(x)=0$ elsewhere we have by $(1.8)$

$$
U(t+h)-U(t)=\int_{0}^{t+h} z(t+h-x) U\{d x\} \sim \frac{C_{\alpha} h}{m(t+h)} \sim C_{\alpha} \frac{h}{m(t)}
$$

as $t \rightarrow \infty$. (That $m(t+h) \sim m(t), t \rightarrow \infty, h$ fixed, follows easily from monotonicity and regular variation of $m$, see Lemma 1.) Thus Theorem 3 is equivalent to Theorem 1 (we use Theorem 1 to prove Theorem 3). Similarly Theorem 4 (with $0<\alpha \leqq \frac{1}{2}$ ) is equivalent to Theorem 2 .

For a generalization of (1.8) to nonintegrable but regularly varying $z$ see §2(iii).

$\$ \S 3-8$ of this paper are concerned with the proofs of Theorems $1-4$. In $\$ 9$ we give an application of the special case $\alpha=1$ to obtain some curious limit theorems for the spent and residual waiting times of a renewal process.

2. Notes. (i) Let $m$ and $U$ be defined as in $\S 1$ and let $\hat{m}$ and $\hat{U}$ be their Laplace transforms:

$$
\hat{m}(\lambda)=\int_{0}^{\infty} e^{-\lambda x}(1-F(x)) d x, \quad \hat{U}(\lambda)=\int_{0}^{\infty} e^{-\lambda x} U\{d x\} .
$$

If in addition $\hat{F}$ is the transform of $F$ then by (1.1) and (1.3)

$$
\hat{m}(\lambda)=\frac{1-\hat{F}(\lambda)}{\lambda}, \quad \hat{U}(\lambda)=\frac{1}{1-\hat{F}(\lambda)}
$$

and hence $\hat{O}(\lambda) \hat{m}(\lambda)=1 / \lambda$. Using this relation and Karamata's Tauberian theorem, $[3$, p. 420], we conclude the following:

THEOREM 5. Let $0 \leqq \alpha \leqq 1$. Each of statements (a) and (b) which follow implies the other and both imply the asymptotic relation (2.1).

(a) $m$ is regularly varying with exponent $1-\alpha$.

(b) $U$ is regularly varying with exponent $\alpha$.

$$
U(t) \sim[\Gamma(\alpha+1) \Gamma(2-\alpha)]^{-1}(t / m(t)) .
$$

By Lemma 1 statement (a) is true when $F$ satisfies (1.2). (The converse is also true provided $\alpha \neq 1$; if (a) is true for some $0 \leqq \alpha<1$, then (1.2) holds for some slowly 
varying $L$, cf. [3, p. 422].) When $\alpha \neq 1$ in (1.2) we see as in the remark following Theorem 2 that $(2.1)$ is equivalent to

$$
U(t) \sim \frac{\sin \pi \alpha}{\pi \alpha} \frac{t^{\alpha}}{L(t)}, \quad t \rightarrow \infty,
$$

(when $\alpha=0,(\sin \pi \alpha) / \pi \alpha \equiv 1$ ). For a proof of $(2.2)$ when $0<\alpha<1$ cf. [3, p. 446]. See also Teugels [10]. When $\frac{1}{2}<\alpha \leqq 1$ (2.1) may also be derived from Theorem 1 (1.4). We shall not do this however. Theorem 1 cannot be proved from (2.1).

(ii) Let $F$ be an arithmetic distribution on $(0, \infty)$ which we suppose, without loss of generality, has span 1. (A distribution has span $b>0$ if it is concentrated on the multiples of $b$ and $b$ is the largest such number.) The renewal measure $U$ defined by (1.1) is also arithmetic with span 1 . Denote by $f_{n}$ and $u_{n}$ the mass assigned to the integer $n$ by $F$ and $U$. If $F$ satisfies (1.2), i.e.,

$$
1-F(n)=\sum_{n+1}^{\infty} f_{k}=n^{-\alpha} L(n)
$$

for some $0<\alpha<1$ and slowly varying $L$, then (Lamperti-Garsia, 1962) for $\frac{1}{2}<\alpha<1$

$$
\lim _{n \rightarrow \infty} n^{1-\alpha} L(n) u_{n}=\frac{\sin \pi \alpha}{\pi}
$$

while for $0<\alpha \leqq \frac{1}{2}$ the lim must be replaced by lim inf. However (2.3) does hold when $0<\alpha \leqq \frac{1}{2}$ provided the limit is taken excluding a set of intergers having density 0 .

These authors did not consider the case $\alpha=1$ (nor, for that matter, $\alpha=0$ ). The appropriate and true conclusion for $\alpha=1$ is

$$
\lim _{n \rightarrow \infty} m(n) u_{n}=1
$$

where, as before,

$$
m(n)=\int_{0}^{n}(1-F(x)) d x=\sum_{k=1}^{n} \sum_{j=k}^{\infty} f_{j} \sim \sum_{1}^{n} j f_{j}, \quad n \rightarrow \infty .
$$

The proof of (2.3) and (2.4) starts with the following representation for $u_{n}$ (see [5] or [8, pp. 98-99]): let $\phi(\theta)=\sum f_{k} e^{i k \theta}$ and put $W(\theta)=\operatorname{Re}[1-\phi(\theta)]^{-1}$ then provided $F$ has an infinite mean

$$
u_{n}=\frac{1}{\pi} \operatorname{Re} \int_{0}^{\pi} \frac{e^{-i n \theta}}{1-\phi(\theta)} d \theta=\frac{2}{\pi} \int_{0}^{\pi} W(\theta) \cos n \theta d \theta
$$

for $n \geqq 1$. (When the mean $\mu$ is finite (2.5) holds with $u_{n}$ replaced by $u_{n}-1 / \mu$.) The lack of a similar formula for $U(t+h)-U(t)$ when $F$ is nonarithmetic constitutes the chief difficulty in the proof of Theorem 1.

Here is a brief proof of (2.4): from (2.5)

$$
\frac{\pi}{2} u_{n}=\left(\int_{0}^{B / n}+\int_{B / n}^{\pi / 2}\right) W(\theta) \cos n \theta d \theta=J_{1}+J_{2} .
$$


As in the latter part of the proof of Theorem 1, see (5.10) and (5.11), we get

$$
\lim _{n \rightarrow \infty} m(n) J_{1}=\pi / 2, \quad \limsup _{n \rightarrow \infty} m(n)\left|J_{1}\right|=O(1 / B) .
$$

(The first limit follows directly from Lemma 4, $\alpha=1$.) Hence

$$
\lim _{n \rightarrow \infty} m(n) u_{n}=\lim _{B \rightarrow \infty} \lim _{n \rightarrow \infty}(2 / \pi) m(n)\left(J_{1}+J_{2}\right)=1 .
$$

J. A. Williamson [11] has extended the results of Lamperti and Garsia [5] to include distributions not necessarily restricted to the positive integers nor to 1-dimension. He does not, however, consider nonarithmetic distributions. He also gives examples showing that (2.3) and its generalization to $d$-dimensions cannot hold when $\alpha \leqq d / 2$ without making further assumptions on $F$. In this connection, see also $[5, \S 3.4]$.

(iii) Suppose the positive function $z$ on $(0, \infty)$ is nondecreasing and regularly varying with exponent $\beta>0$. Consider the integral

$$
U^{*} z(t)=\int_{0}^{t} z(t-x) U\{d x\}=\int_{0}^{1} z(t(1-y)) U\{t d y\} .
$$

By Theorem $5 U(t y) / U(t) \rightarrow y^{\alpha}$ and it follows that the measure $U\{t d y\} / U(t)$ converges weakly as $t \rightarrow \infty$ to the measure with density $\alpha y^{\alpha-1}$. Furthermore

$$
f_{t}(y)=z(t(1-y)) / z(t) \rightarrow(1-y)^{\beta}, \quad t \rightarrow \infty
$$

and the convergence is uniform in $y, 0 \leqq y \leqq 1$, since each $f_{t}(y)$ is monotone in $y$ and the limit function $(1-y)^{\beta}$ is continuous. We see therefore that

$$
\frac{U^{*} z(t)}{z(t) U(t)}=\int_{0}^{1} \frac{z(t(1-y))}{z(t)} \cdot \frac{U\{t d y\}}{U(t)} \rightarrow \alpha \int_{0}^{1}(1-y)^{\beta} y^{\alpha-1} d y
$$

as $t \rightarrow \infty$. Now $t z(t) \sim(1+\beta) \int_{0}^{t} z(x) d x$ by Karamata's theorem on regular variation, [3, p. 273]. Hence using (2.1) we see that (2.7) may be put in the equivalent form

$$
\int_{0}^{t} z(t-x) U\{d x\} \sim \frac{D(\alpha, \beta)}{m(t)} \int_{0}^{t} z(x) d x, \quad t \rightarrow \infty
$$

where

$$
D(\alpha, \beta)=\frac{\alpha(1+\beta)}{\Gamma(1+\alpha) \Gamma(2-\alpha)} \cdot \int_{0}^{1}(1-y)^{\beta} y^{\alpha-1} d y=\frac{\Gamma(2+\beta)}{\Gamma(\alpha+\beta+1) \Gamma(2-\alpha)} .
$$

Notice that the proof of (2.7) and (2.8) did not depend on the renewal nature, (1.1), of $U ;(2.8)$ remains true when $U>0$ is any nondecreasing function regularly varying with exponent $\alpha, 0<\alpha \leqq 1$, and $m$ is any function satisfying (2.1).

J. Teugels [10] gave a proof of (2.8) when $z>0$ is nonincreasing and regularly varying with exponent $\beta$ where $-1<\beta \leqq 0$. The proof is much complicated by the fact that convergence in (2.6) is no longer uniform: when $\beta<0$ the function 
$(1-y)^{\beta}$ is not bounded at $y=1$. (Teugels imposes a supplementary and rather technical condition on $U$, in addition to regular variation, which seems to me to be unnecessary; compare the proof in Feller [3, p. 447], of a result where similar problems arise.) Again the proof makes no use of the renewal properties of $U$.

The regular variation of $z$ with exponent $\beta>-1$ and to a lesser extent the monotonicity of $z$ is clearly essential to the proof of (2.8). In particular, the condition $\beta>-1$ cannot be dropped. When $\beta>-1$, the integral $\int_{0}^{t} z(x) d x$ occurring in (2.8) diverges to $\infty$ as $t \rightarrow \infty$, while for $\beta<-1, \int_{A}^{\infty} z(x) d x$ is finite for all large enough $A$. In this case, $\beta<-1$, Theorem $3, \S 1$, usually applies and leads to results directly opposed to (2.8). For example, let $z(t)=t^{-5}, t>1$ and $z(t)=1, t \leqq 1$ ( $z$ is regularly varying with exponent $\beta=-5$ ). Then $\int_{0}^{\infty} z(x) d x=5 / 4$ and, provided $\alpha>\frac{1}{2}$, Theorem 3 gives $m(t) U^{*} z(t) \rightarrow C_{\alpha} 5 / 4<\infty$ as $t \rightarrow \infty$. On the other hand, if (2.8) were true we would get $m(t) U^{*} z(t) \rightarrow D(\alpha,-5) 5 / 4=\infty$.

One last remark. As noted before, one could prove Theorem 5 from Theorem 1 (and Lemma 1) at least for $\frac{1}{2}<\alpha \leqq 1$. Since (2.8) depends only on Theorem 5 for the regular variation of $U$ and since Theorem 3 is equivalent to Theorem 1, we see that (2.8) could be derived from Theorem 3, at least in principle, when the only data given, besides the function $z$, is that $U$ is the renewal function of a distribution $F$ of the form (1.2). In no way, however, can Theorem 3 be proved from (2.8).

(iv) The classical "strong" and "weak" renewal theorems assert respectively

$$
U(t+h)-U(t) \rightarrow h / \mu \quad(h>0)
$$

$$
(1 / t) U(t) \rightarrow 1 / \mu
$$

as $t \rightarrow \infty$, for any (nonarithmetic) distribution $F$ on $(0, \infty)$ with mean $\mu \leqq \infty$ $(1 / \mu$ is interpreted as 0 when $\mu=\infty)$. Since $m(t) \rightarrow \mu$ as $t \rightarrow \infty$ we may rewrite (2.9) and (2.10) as

$$
U(t+h)-U(t) \sim h / m(t), \quad U(t) \sim t / m(t)
$$

provided $\mu<\infty$. Thus apart from the constant $C_{\alpha}$ in (1.4) and $[\Gamma(\alpha+1) \Gamma(2-\alpha)]^{-1}$ $=C_{\alpha} / \alpha$ in (2.1), Theorems 1 and 5 are the natural generalizations of these classical theorems.

(v) It should be pointed out that when $\alpha=1$ in (1.2), i.e., if $F$ has the form $1-F(t)=L(t) / t$ for some slowly varying $L$, then $F$ may or may not have a finite mean. For an example when $\mu<\infty$ consider $L(t)=[\log (t+2)]^{-3} \sim(\log t)^{-3}$. For $\mu=\infty$, consider $L(t) \sim$ const $>0$.

As noted in (iv), the classical theorems already imply Theorem 1 (and 5) when $\mu<\infty$. Hence we shall assume from now on that $\mu=\infty$ when $\alpha=1$ in (1.2).

3. Properties of distributions satisfying (1.2). Let $F$ be of the form (1.2) (when $\alpha=1$ we assume in addition that $F$ have infinite expectation, see \$2). Let $\phi$ be the characteristic function of $F$ :

$$
\phi(\theta)=\int_{0}^{\infty} e^{i x \theta} F\{d x\}
$$


LEMMA 1. The function $m$ defined by (1.3) is regularly varying with exponent $1-\alpha$, and as $t \rightarrow \infty$

$$
t(1-F(t)) / m(t)=t^{1-\alpha} L(t) / m(t) \rightarrow 1-\alpha .
$$

We shall need the following immediate consequence of Lemma $1:$ let $\eta>0$, then provided $\alpha>1 / 2$ and $B>0$,

$$
\lim _{t \rightarrow \infty} t^{-1} m^{2}(t) \int_{\eta}^{t / B} m^{-2}(x) d x=\left[(2 \alpha-1) B^{2 \alpha-1}\right]^{-1} .
$$

NoTE. The restriction to $\alpha>1 / 2$ in (3.2) partly explains the failure (at least of the proof) of Theorems 1 and 3 when $\alpha \leqq 1 / 2$. See equation (5.11).

Proof. This lemma is a direct consequence of Karamata's theorem on regularly varying functions, see Feller [3, p. 273]. The relation (3.2) likewise follows from this theorem. To see this, define $Z(x)=m^{-2}(x)$ for $x \geqq \eta, Z(x)=0,0 \leqq x<\eta$. Since $m$ is regularly varying with exponent $1-\alpha, Z$ varies regularly with exponent $-2(1-\alpha)=2 \alpha-2$. Hence, according to the theorem,

$$
\lim _{t \rightarrow \infty} \frac{t Z(t)}{\int_{0}^{t} Z(x) d x}=\lim _{t \rightarrow \infty} \frac{(t / B) Z(t / B)}{\int_{0}^{t / B} Z(x) d x}=1+2 \alpha-2=2 \alpha-1 .
$$

But $Z(t / B) \sim(1 / B)^{2 \alpha-2} Z(t), t \rightarrow \infty$ (by definition of regular variation). Therefore

$$
\int_{\eta}^{t / B} m^{-2}(x) d x \sim(2 \alpha-1)^{-1}(t / B) Z(t / B) \sim t m^{-2}(t) /(2 \alpha-1) B^{2 \alpha-1}
$$

as $t \rightarrow \infty$ which proves (3.2).

LEMMA 2. As $\theta \rightarrow 0+$

$$
1-\phi(\theta) \sim e^{-i \pi \alpha / 2} \Gamma(2-\alpha) \theta m(1 / \theta) \quad(\alpha \neq 0) .
$$

When $\alpha=1$ we have in addition to (3.3)

$$
\operatorname{Re}(1-\phi(\theta)) \sim \frac{1}{2} \pi \theta L(1 / \theta), \quad \theta \rightarrow 0+.
$$

Proof. Suppose $0<\alpha<1$. Then by (3.1) $m(1 / \theta) \sim(1-\alpha)^{-1} \theta^{\alpha-1} L(1 / \theta), \theta \rightarrow 0+$. Since $\Gamma(2-\alpha) /(1-\alpha)=\Gamma(1-\alpha)$ we see that (3.3) is equivalent to

$$
1-\phi(\theta) \sim e^{-i \pi \alpha / 2} \Gamma(1-\alpha) \theta^{\alpha} L(1 / \theta), \quad \theta \rightarrow 0+.
$$

Stated in this form (3.3) is well known so we omit the proof. See Garsia and Lamperti [5], or Feller [3, Problems 12 and 13, p. 562]. (There is a slight misprint in the latter reference.)

When $\alpha=1,(3.3)$ and (3.4) do not seem to be as well known. Here then is a brief proof. For any $A, \theta>0$, write

$$
1-\phi(\theta)=\left(\int_{0}^{A / \theta}+\int_{A / \theta}^{\infty}\right)\left(1-e^{i y \theta}\right) F\{d y\}=J_{1}+J_{2}
$$


then

$$
\begin{aligned}
\left|J_{2}\right| & =\left|\int_{A / \theta}^{\infty}\left(1-e^{i y \theta}\right) F\{d y\}\right| \leqq 2(1-F(A / \theta)), \\
J_{1} & =\int_{0}^{A / \theta}\left(1-e^{i y \theta}\right) F\{d y\}=-\left(1-e^{i A}\right)(1-F(A / \theta))-i \int_{0}^{A} e^{i x}(1-F(x / \theta)) d x .
\end{aligned}
$$

But $1-F(t)=L(t) / t$ with $L$ slowly varying. Hence

$$
1-\phi(\theta)=O\left(\frac{\theta L(A / \theta)}{A}\right)-i \int_{0}^{A} e^{i x}(1-F(x / \theta)) d x .
$$

(The bound in the 0 term is $\leqq 4$ in magnitude.)

We prove (3.3) first. From (3.1) and slow variation of $L$ we get

$$
L(A / \theta) \sim L(1 / \theta)=o(m(1 / \theta)), \quad \theta \rightarrow 0+.
$$

Hence from (3.6)

$$
\lim _{\theta \rightarrow 0+} \frac{1-\phi(\theta)}{\theta m(1 / \theta)}=-i \lim _{\theta \rightarrow 0+} \int_{0}^{A} e^{i x}\left(\frac{1-F(x / \theta)}{\theta m(1 / \theta)}\right) d x
$$

provided the latter limit exists. Now by Lemma $1 \mathrm{~m}$ is slowly varying (三 regularly varying with exponent 0 ); also $m(0)=0$. Hence, the measure $Q_{\theta}$ on $[0, A]$ with distribution function $Q_{\theta}(y)=m(y / \theta) / m(1 / \theta)$ converges weakly as $\theta \rightarrow 0+$ to the measure which assigns unit mass to the origin. Whence, for any continuous $g$ on $[0, A]$

$$
\int_{0}^{A} g(x) Q_{\theta}\{d x\}=\int_{0}^{A} g(x)\left(\frac{1-F(x / \theta)}{\theta m(1 / \theta)}\right) d x \rightarrow g(0)
$$

as $\theta \rightarrow 0+$. Taking $g(x)=e^{i x}$ we see that the right-hand side of (3.7) equals $-i$. This proves (3.3).

NoTE. The preceding proof requires only minor changes to apply in the case $0<\alpha<1$. In particular, a term $O\left(1 / A^{\alpha}\right)$ must be added to the right side of (3.7); also $Q_{\theta}$ converges to the measure with density $(1-\alpha) x^{-\alpha}$. In (3.7) one lets $\theta \rightarrow 0+$ followed by $A \rightarrow \infty$. The remainder of the proof is then an evaluation of an improper integral.

To prove (3.4), take real parts in (3.6). Then

$$
\frac{\operatorname{Re}(1-\phi(\theta))}{\theta L(1 / \theta)}=O\left(\frac{1}{A}\right)+\int_{0}^{A} \frac{\sin x}{x} \cdot \frac{L(x / \theta)}{L(1 / \theta)} d x .
$$

(The bound in the 0 term is $\leqq 8$ for all $0<\theta \leqq \theta_{A}$ sufficiently small.) Letting $\theta \rightarrow 0+$ and then $A \rightarrow \infty$ we see that

$$
\lim _{\theta \rightarrow 0} \frac{\operatorname{Re}(1-\phi(\theta))}{\theta L(1 / \theta)}=\lim _{A \rightarrow \infty} \lim _{\theta \rightarrow 0} \int_{0}^{A} \frac{\sin x}{x} \cdot \frac{L(x / \theta)}{L(1 / \theta)} d x
$$


provided the iterated limit exists. Since $L$ is slowly varying, we get from the Karamata theorem mentioned earlier

$$
\int_{0}^{t} L(u) d u \sim t L(t), \quad t \rightarrow \infty
$$

Hence, for every $y \geqq 0$,

$$
\lim _{\theta \rightarrow 0} \int_{0}^{y} \frac{L(x / \theta)}{L(1 / \theta)} d x=\lim _{\theta \rightarrow 0} \frac{\theta}{L(1 / \theta)} \int_{0}^{y / \theta} L(u) d u=y .
$$

That is, the measure with density $L(x / \theta) / L(1 / \theta), x \geqq 0$, converges weakly as $\theta \rightarrow 0$ to Lebesgue measure. Hence for any continuous function $f$ and any compact interval $[0, A]$, say,

$$
\lim _{\theta \rightarrow 0} \int_{0}^{A} f(x)\left(\frac{L(x / \theta)}{L(1 / \theta)}\right) d x=\int_{0}^{A} f(x) d x .
$$

Letting $f(x)=(\sin x) / x$ and returning to (3.8) we have

$$
\lim _{\theta \rightarrow 0+} \frac{\operatorname{Re}(1-\phi(\theta))}{\theta L(1 / \theta)}=\lim _{A \rightarrow \infty} \int_{0}^{A} \frac{\sin x}{x} d x=\frac{\pi}{2},
$$

which proves (3.4).

For the purposes of the next two lemmas put

$$
W(x)=\operatorname{Re}\left(\frac{1}{1-\phi(x)}\right)=\frac{\operatorname{Re}(1-\phi(x))}{|1-\phi(x)|^{2}} .
$$

Note that $W$ is positive since $\operatorname{Re}(1-\phi(x))=\int_{0}^{\infty}(1-\cos x t) F\{d t\}>0$, and symmetric: $W(-x)=W(x)$. Also, $W$ is unbounded (hence undefined) at all $x$ for which $\phi(x)=1$ (in particular at $x=0$ ); at all other $x W$ is continuous.

LEMMA 3. As $\theta \rightarrow 0+$

$$
\int_{0}^{\theta} W(x) d x \sim \frac{\cos (\pi \alpha / 2)}{(1-\alpha) \Gamma(2-\alpha)} \cdot \frac{1}{m(1 / \theta)}
$$

When $\alpha=1$ the constant on the right is replaced by

$$
\frac{\pi}{2}\left(=\lim _{\alpha \rightarrow 1} \frac{\cos (\pi \alpha / 2)}{(1-\alpha) \Gamma(2-\alpha)}\right) .
$$

REMARK. The integrability of $W$ over bounded intervals containing the origin is, of course, part of the conclusion. This fact, however, is true for any distribution on $(0, \infty)$ (and for some distributions on the entire line); see [3, p. 578].

Proof. A simple calculation using (3.9) and the asymptotic relations (3.3), (3.4) and (3.5) gives

$$
W(x) \sim \frac{k_{\alpha} L(1 / x)}{x^{2-\alpha} m^{2}(1 / x)}, \quad x \rightarrow 0+,
$$


where $k_{\alpha}$ is the constant occurring on the right in (3.10) $\left(k_{1}=\pi / 2\right)$. Next note that the function $1 / m(1 / x), x>0$ is absolutely continuous on any interval bounded away from 0 and $\infty$. So, by the chain rule and (1.2)

$$
\frac{d}{d x}\left(\frac{1}{m(1 / x)}\right)=\frac{1-F(1 / x)}{x^{2} m^{2}(1 / x)}=\frac{L(1 / x)}{x^{2-\alpha} m^{2}(1 / x)}
$$

for almost all $x$. (The exceptional set is at most countable.)

Consider $0<\varepsilon<1$ fixed but arbitrary. By (3.11) there is a $\lambda=\lambda(\varepsilon)>0$ such that

$$
W(x) \gtrless(1 \pm \varepsilon) k_{\alpha} \cdot \frac{L(1 / x)}{x^{2-\alpha} m^{2}(1 / x)}
$$

whenever $0<x \leqq \lambda$. Integrating these inequalities from $x=\delta$ to $x=\theta$ and using (3.12) yields

$$
\int_{\delta}^{\theta} W(x) d x \lessgtr(1 \pm \varepsilon) k_{\alpha}\left(\frac{1}{m(1 / \theta)}-\frac{1}{m(1 / \delta)}\right)
$$

for $0<\delta \leqq \theta \leqq \lambda$. Now let $\delta \rightarrow 0$, then $m(1 / \delta) \rightarrow \infty(\mu=\infty$ recall $)$, hence

$$
(1-\varepsilon) \frac{k_{\alpha}}{m(1 / \theta)}<\int_{0}^{\theta} W(x) d x<(1+\varepsilon) \frac{k_{\alpha}}{m(1 / \theta)}
$$

whenever $0<\theta \leqq \lambda$. This concludes the proof.

By Lemmas 1 and 3, as $t \rightarrow \infty$

$$
\frac{m(t)}{t} \int_{0}^{\theta} W(y / t) d y=m(t) \int_{0}^{\theta / t} W(x) d x \rightarrow k_{\alpha} \theta^{1-\alpha}
$$

for all $\theta>0$ and it follows that the measure with density $q_{t}(y)=(m(t) / t) W(y / t)$ converges weakly as $t \rightarrow \infty$ to a measure which when $\alpha=1$ is concentrated at the origin with total mass $k_{1}=\pi / 2$ and when $0<\alpha<1$ is absolutely continuous with density $(1-\alpha) k_{\alpha} y^{-\alpha}$. Denote the limit measure by $E_{\alpha}$. Then for any function $f$ continuous on a compact interval, $[0, B]$, say,

$$
m(t) \int_{0}^{B / t} f(t \theta) W(\theta) d \theta=\int_{0}^{B} f(y) q_{t}(y) d y \rightarrow \int_{0}^{B} f(y) E_{\alpha}\{d y\}, \quad t \rightarrow \infty
$$

Taking $f(y)=\cos y$ we have

Lemma 4. Let $W$ be given by (3.9). Then for any $B>0$

$$
\begin{aligned}
\lim _{t \rightarrow \infty} m(t) \int_{0}^{B / t} W(\theta) \cos t \theta d \theta & =\frac{\cos (\pi \alpha / 2)}{\Gamma(2-\alpha)} \int_{0}^{B} \frac{\cos y}{y^{\alpha}} d y, & & \alpha \neq 1, \\
& =\pi / 2, & & \alpha=1 .
\end{aligned}
$$

LEMMA 5. (i) For all $\theta_{1} \neq \theta_{2}$

$$
\left|\phi\left(\theta_{2}\right)-\phi\left(\theta_{1}\right)\right| \leqq 2\left|\theta_{2}-\theta_{1}\right| m\left(1 /\left|\theta_{2}-\theta_{1}\right|\right)
$$


(ii) If $F$ is nonarithmetic, then for each $A>0$, there is a number $k>0$, which may depend on $A$, such that

$$
\theta m(1 / \theta) \leqq k|1-\phi(\theta)| \text { for } 0<\theta \leqq A
$$

If $F$ is arithmetic with span $h,(3.16)$ is true provided $A<2 \pi / h=$ period of $\phi$.

Proof. (i) Fix $B>0$. Then

$$
\begin{aligned}
\left|\phi\left(\theta_{2}\right)-\phi\left(\theta_{1}\right)\right| & =\left|\left(\int_{0}^{B}+\int_{B}^{\infty}\right)\left(e^{i x \theta_{2}}-e^{i x \theta_{1}}\right) F\{d x\}\right| \\
& \leqq \int_{0}^{B}\left|e^{i x \theta_{2}}-e^{i x \theta_{1}}\right| F\{d x\}+2(1-F(B)) \\
& \leqq\left|\theta_{2}-\theta_{1}\right| \int_{0}^{B} x F\{d x\}+2(1-F(B)) .
\end{aligned}
$$

But $0 \leqq \int_{0}^{B} x F\{d x\}=m(B)-B(1-F(B))$ by (1.3). Hence setting $B=\left|\theta_{2}-\theta_{1}\right|^{-1}$ we get $\left|\phi\left(\theta_{2}\right)-\phi\left(\theta_{1}\right)\right| \leqq B^{-1}[m(B)-B(1-F(B))]+2(1-F(B))=B^{-1} m(B)+1-F(B) \leqq$ $2 B^{-1} m(B)$ which proves (3.15). (Note that (1.2) was not used; (3.15) holds for any $F$ on $[0, \infty)$.)

(ii) If $F$ is nonarithmetic then $|1-\phi(\theta)|>0$ for all $\theta \neq 0$. By Lemma 2 as $\theta \rightarrow 0+$

$$
\theta m(1 / \theta) /|1-\phi(\theta)| \rightarrow 1 / \Gamma(2-\alpha)
$$

and it follows that the function

$$
\begin{aligned}
\beta(\theta) & =\theta m(1 / \theta)|1-\phi(\theta)|^{-1}, & & \theta \neq 0 \\
& =(\Gamma(2-\alpha))^{-1}, & & \theta=0
\end{aligned}
$$

is continuous on $[0, A]$. Taking $k=\max \{\beta(\theta): 0 \leqq \theta \leqq A\}$ gives (3.16).

4. An inversion formula for the renewal measure. Define the symmetric renewal measure

$$
V\{I\}=\frac{1}{2}(U\{I\}+U\{-I\})
$$

where $U$ is given by (1.1) and $-I=\{x:-x \in I\}$. In this section we establish the following

Formula. Suppose $F$ is nonarithmetic and has an infinite mean. Then for any continuous function $g$ with compact support whose Fourier transform

$$
\gamma(x)=\int_{-\infty}^{\infty} e^{i x \theta} g(\theta) d \theta
$$

satisfies

$$
\gamma(x)=O\left(1 / x^{2}\right), \quad|x| \rightarrow \infty
$$

we have

$$
\int_{-\infty}^{\infty} e^{-i x \lambda} \gamma(x) V\{t+d x\}=\int_{-\infty}^{\infty} e^{-i t \theta} g(\theta+\lambda) \operatorname{Re}\left(\frac{1}{1-\phi(\theta)}\right) d \theta
$$


for all real $\lambda$ and $t$. Here, as elsewhere, $\phi$ is the characteristic function of $F$. Note that the integral on the right in (4.3) only extends over a bounded interval. For examples of $g$ and $\gamma$ see $\S 5$.

LEMMA 6. Let $\gamma$ be any continuous function satisfying (4.2). Then for every $t$ the integral

$$
\int_{-\infty}^{\infty}|\gamma(x-t)| V\{d x\}
$$

is finite.

Proof. Since $\int_{-1}^{1}|\gamma(x-t)| V\{d x\}<\infty$ and since $|\gamma(x-t)|$ is bounded by a constant (which may depend on $t$ but not $x$ ) times $1 / x^{2}$, it suffices to show

$$
\int_{|x| \geqq 1} \frac{1}{x^{2}} V\{d x\}=\int_{1}^{\infty} \frac{1}{x^{2}} U\{d x\}<\infty .
$$

From (2.10) it follows that $U(x) \leqq k_{1} x$ for some constant $k_{1}<\infty$ and all $x \geqq 1$. Therefore integrating by parts in (4.4) we get

$$
\begin{aligned}
\int_{1}^{\infty} \frac{1}{x^{2}} U\{d x\} & =\lim _{A \rightarrow \infty}\left(\frac{U(A)}{A^{2}}-U(1)+2 \int_{1}^{A} \frac{U(x)}{x^{3}} d x\right) \\
& =-U(1)+2 \int_{1}^{\infty} \frac{U(x)}{x^{3}} d x \leqq 2 k_{1} \int_{1}^{\infty} \frac{1}{x^{2}} d x<\infty
\end{aligned}
$$

which proves (4.4) and the lemma.

For $0 \leqq s<1$ let $V_{s}$ be the finite symmetric measure

$$
V_{s}\{d x\}=\frac{1}{2} \sum_{n=0}^{\infty} s^{n}\left(F^{n^{*}}\{d x\}+F^{n^{*}}\{-d x\}\right)
$$

and note that

$$
V_{s}\{I\} \uparrow V\{I\} \quad \text { as } s \uparrow 1
$$

for every measurable $I$ bounded or not.

Since

$$
\phi(-\theta)=\overline{\phi(\theta)}
$$

we have

$$
\int_{-\infty}^{\infty} e^{i x \theta} V_{s}\{d x\}=\frac{1}{2} \sum_{0}^{\infty} s^{n}\left(\phi^{n}(\theta)+\phi^{n}(-\theta)\right)=\operatorname{Re}\left(\frac{1}{1-s \phi(\theta)}\right)
$$

and an application of Fubini's theorem gives

$$
\int_{-\infty}^{\infty} \gamma(x) V_{s}\{d x\}=\int_{-\infty}^{\infty} g(\theta) \operatorname{Re}\left(\frac{1}{1-s \phi(\theta)}\right) d \theta \quad(0 \leqq s<1)
$$

for any (Lebesgue) integrable function $g$ with $\gamma$ given by (4.1). Replacing $g$ by

$$
g_{1}(\theta)=e^{-i t \theta} g(\theta+\lambda)
$$


and $\gamma$ by

$$
\gamma_{1}(x)=\int_{-\infty}^{\infty} e^{i x \theta} g_{1}(\theta) d \theta=e^{-i \lambda(x-t)} \gamma(x-t)
$$

we get

$$
\int_{-\infty}^{\infty} e^{-i \lambda(x-t)} \gamma(x-t) V_{s}\{d x\}=\int_{-\infty}^{\infty} e^{-i t \theta} g(\theta+\lambda) \operatorname{Re}\left(\frac{1}{1-s \phi(\theta)}\right) d \theta
$$

LEMMA 7. For any continuous function $h$ with compact support

$$
\lim _{s \rightarrow 1-} \int_{-\infty}^{\infty} h(\theta) \operatorname{Re}\left(\frac{1}{1-s \phi(\theta)}\right) d \theta=\int_{-\infty}^{\infty} h(\theta) \operatorname{Re}\left(\frac{1}{1-\phi(\theta)}\right) d \theta
$$

provided $F$ is nonarithmetic and has infinite expectation.

Proof. We base the proof on the following proposition due to Feller and Orey [4]:

Proposition. The measure whose density is

$$
\frac{1}{1+\theta^{2}} \operatorname{Re}\left(\frac{1}{1-s \phi(\theta)}\right)
$$

converges weakly and in variation to a finite measure as $s \rightarrow 1-$. In every interval excluding the origin the limit measure is automatically absolutely continuous with density given by

$$
\frac{1}{1+\theta^{2}} \operatorname{Re}\left(\frac{1}{1-\phi(\theta)}\right)
$$

If $\beta$ is the mass assigned to the origin by the limit then $\beta=\pi / \mu>0$ when $\mu$ (the mean of $F$ ) is finite and $\beta=0$ in case $\mu=\infty$.

We omit the proof. (Besides the Feller-Orey paper, see also Breimann [1, p. 221], and Feller [3, p. 578].) The proposition implies, among other things, that

$$
\lim _{s \rightarrow 1-} \int_{-\infty}^{\infty} \frac{f(\theta)}{1+\theta^{2}} \operatorname{Re}\left(\frac{1}{1-s \phi(\theta)}\right) d \theta=\beta f(0)+\int_{-\infty}^{\infty} \frac{f(\theta)}{1+\theta^{2}} \operatorname{Re}\left(\frac{1}{1-\phi(\theta)}\right) d \theta
$$

for every continuous function $f$ with compact support. In our case $\beta=0$, and (4.7) follows by setting $f(\theta)=\left(1+\theta^{2}\right) h(\theta)$.

Proof of formula (4.3). The very strong convergence (4.5) of the measures $V_{s}$ to $V$ implies

$$
\lim _{s \rightarrow 1-} \int_{-\infty}^{\infty} f(x) V_{s}\{d x\}=\int_{-\infty}^{\infty} f(x) V\{d x\}
$$

for every $f$ integrable with respect to $V$. (In fact, if $f$ is nonnegative the integral on the left is nondecreasing as a function of $s$ and one can show (4.8) holds even if $f$ is not integrable.) 
Suppose now $g$ and $\gamma$ satisfy (4.1) and (4.2) with $g$ continuous and vanishing off a compact set. Then by Lemma 6

$$
e^{-i \lambda(x-t)} \gamma(x-t)
$$

is integrable with respect to $V\{d x\}$ for every $t$ and $\lambda$. Hence by (4.6) and (4.8)

$$
\begin{aligned}
\int_{-\infty}^{\infty} e^{-\imath \lambda x} \gamma(x) V\{t+d x\} & \equiv \int_{-\infty}^{\infty} e^{-t \lambda(x-t)} \gamma(x-t) V\{d x\} \\
& =\lim _{s \rightarrow 1-} \int_{-\infty}^{\infty} e^{-i t \theta} g(\theta+\lambda) \operatorname{Re}\left(\frac{1}{1-s \phi(\theta)}\right) d \theta .
\end{aligned}
$$

Formula (4.3) now follows from Lemma 7.

\section{Proof of Theorem 1.}

$1^{\circ}$. Introduce measures $\mu_{t}, t>0$, by

$$
\mu_{t}\{I\}=2 m(t) V\{I+t\}=m(t)(U\{I+t\}+U\{-I-t\})
$$

where $I$ is measurable and $I+t=\{x: x-t \in I\}$. Since $U$ is concentrated on $[0, \infty)$ it follows by taking $I=[0, h]$ in (5.1) that

$$
U(t+h)-U(t)=(1 / m(t)) \mu_{t}\{I\} .
$$

Therefore to prove Theorem 1 it suffices to show

$$
\mu_{t}\{I\} \rightarrow C_{\alpha}|I|, \quad t \rightarrow \infty,
$$

for every bounded interval $I$ where $|I|$ denotes the length of $I$ and

$$
C_{\alpha}=[\Gamma(\alpha) \Gamma(2-\alpha)]^{-1} .
$$

For each $a>0$ put $\gamma_{a}(0)=1$ and

$$
\gamma_{a}(x)=2(1-\cos (a x)) / a^{2} x^{2} .
$$

LEMMA 8. Let $\left\{\mu_{t}\right\}, t>0$, be a family of measures such that $\mu_{t}\{I\}<\infty$ for every compact set $I$ and all $t$. Suppose for some constant $C$

$$
\lim _{t \rightarrow \infty} \int_{-\infty}^{\infty} e^{-i \lambda x} \gamma_{a}(x) \mu_{t}\{d x\}=C \int_{-\infty}^{\infty} e^{-i \lambda x} \gamma_{a}(x) d x
$$

for every $a>0$ and all real $\lambda$. Then $C^{-1} \mu_{t}$ converges weakly to Lebesgue measure: $\mu_{t}\{I\} \rightarrow C|I|$ for every bounded interval $I$.

(We defer the proof until §6.)

Now $\gamma_{a}$ is the Fourier transform (4.1) of the function

$$
\begin{aligned}
g_{a}(\theta) & =(1 / a)(1-|\theta| / a), & & \text { when }|\theta| \leqq a \\
& =0, & & \text { when }|\theta|>a .
\end{aligned}
$$

Whence by the Fourier inversion theorem

$$
\int_{-\infty}^{\infty} e^{-i \lambda x} \gamma_{a}(x) d x=2 \pi g_{a}(\lambda)
$$


Clearly we may also apply our inversion formula (4.3) to obtain

$$
\int_{-\infty}^{\infty} e^{-i \lambda x} \gamma_{a}(x) \mu_{t}\{d x\}=2 m(t) \int_{-\infty}^{\infty} e^{-i t \theta} g_{a}(\theta+\lambda) W(\theta) d \theta
$$

where $W(\theta)=\operatorname{Re}[1-\phi(\theta)]^{-1}$. Note that the integral on the right extends from $\theta=-a-\lambda$ to $\theta=a-\lambda$. From (5.6) and (5.7) we see that (5.4) in our case is equivalent to

$$
\lim _{t \rightarrow \infty} m(t) \int_{-\infty}^{\infty} e^{-i t \theta} g_{a}(\theta+\lambda) W(\theta) d \theta=\pi C g_{a}(\lambda)
$$

and, by Lemma 8, the proof of (5.2) (and Theorem 1) will be completed when we establish (5.8), with $C=C_{\alpha}$ for every $a>0$ and all real $\lambda$.

$2^{\circ}$. Let $B>1$ be fixed but otherwise arbitrary, and write the integral in (5.8) as the sum $J_{1}+J_{2}$ where

$$
\begin{aligned}
J_{1}(t, b) & =\int_{-B / t}^{B / t} e^{-i t \theta} g_{a}(\theta+\lambda) W(\theta) d \theta \text { and } \\
J_{2}(t, B) & =\int_{|\theta|>B / t} e^{-i t \theta} g_{a}(\theta+\lambda) W(\theta) d \theta \\
& =\int_{B / t}^{A}\left[e^{-i t \theta} g_{a}(\theta+\lambda)+e^{i t \theta} g_{a}(\theta-\lambda)\right] W(\theta) d \theta, \\
A & =\max \{a+\lambda, a-\lambda\} .
\end{aligned}
$$

(The last integral follows by making the substitution $\theta \rightarrow-\theta$ in the integral $\int_{-\infty}^{-B / t}$, using the evenness of the functions $g_{a}$ and $W$ and noting that $g_{a}$ vanishes outside the interval $(-a, a)$.) We will show

$$
\begin{aligned}
\lim _{t \rightarrow \infty} m(t) J_{1}(t, B) & =g_{a}(\lambda) \frac{2 \cos \pi \alpha / 2}{\Gamma(2-\alpha)} \int_{0}^{B} \frac{\cos x}{x^{\alpha}} d x, & & \alpha \neq 1 \\
& =\pi g_{a}(\lambda), & & \alpha=1
\end{aligned}
$$

and ${ }^{\circ}$

$$
\limsup _{t \rightarrow \infty} m(t)\left|J_{2}(t, B)\right|=O\left(\frac{1}{B^{2 \alpha-1}}\right), \quad \frac{1}{2}<\alpha \leqq 1
$$

which lead directly to (5.8).

$3^{\circ}$. Proof of (5.10). It is clear from (5.5) that

$$
\left|g_{a}\left(\theta_{2}\right)-g_{a}\left(\theta_{1}\right)\right| \leqq\left(1 / a^{2}\right)\left|\theta_{2}-\theta_{1}\right|
$$

for all $\theta_{1}, \theta_{2}$. Hence

$$
\begin{aligned}
m(t)\left|J_{1}(t, B)-g_{a}(\lambda) \int_{-B / t}^{B / t} e^{-i t \theta} W(\theta) d \theta\right| & \leqq m(t) \int_{-B / t}^{B / t}\left|g_{a}(\theta+\lambda)-g_{a}(\lambda)\right| W(\theta) d \theta \\
& \leqq \frac{2 B}{a^{2}} \cdot \frac{m(t)}{t} \int_{0}^{B / t} W(\theta) d \theta=O\left(\frac{1}{t}\right)
\end{aligned}
$$


where the $O(1 / t)$ follows from (3.10) and Lemma 1 . Thus

$$
\begin{aligned}
\lim _{t \rightarrow \infty} m(t) J_{1}(t, B) & =g_{a}(\lambda) \lim _{t \rightarrow \infty} m(t) \int_{-B / t}^{B / t} e^{-i t \theta} W(\theta) d \theta \\
& =2 g_{a}(\lambda) \lim _{t \rightarrow \infty} m(t) \int_{0}^{B / t} W(\theta) \cos t \theta d \theta
\end{aligned}
$$

and (5.10) now follows from Lemma 4.

$4^{\circ}$. Proof of (5.11). Let

$$
\begin{aligned}
& h_{1}(\theta)=e^{-i t \theta} g_{a}(\theta+\lambda)+e^{i t \theta} g_{a}(\theta-\lambda), \\
& h_{2}(\theta)=e^{-i t \theta} g_{a}(\theta+\pi / t+\lambda)+e^{i t \theta} g_{a}(\theta+\pi / t-\lambda) .
\end{aligned}
$$

Then $h_{1}(\theta+\pi / t)=-h_{2}(\theta)$ and making the change of variables $\theta \rightarrow \theta+\pi / t$ in (5.9) gives

$$
J_{2}(t, B)=\int_{B / t}^{A} h_{1}(\theta) W(\theta) d \theta=\int_{(B-\pi) / t}^{A}-h_{2}(\theta) W(\theta+\pi / t) d \theta
$$

(note that the integrand in the last written integral vanishes for $A-\pi / t \leqq \theta$ ). Adding these integrals we get

(5.13) $2 J_{2}=-\int_{(B-\pi) / t}^{B / t} h_{2}(\theta) W\left(\theta+\frac{\pi}{t}\right) d \theta+\int_{B / t}^{A}\left[h_{1}(\theta) W(\theta)-h_{2}(\theta) W\left(\theta+\frac{\pi}{t}\right)\right] d \theta$.

Now $\left|h_{j}(\theta)\right| \leqq 2 / a$ and from (5.12) we have

$$
\left|h_{1}(\theta)-h_{2}(\theta)\right| \leqq\left|g_{a}(\theta+\lambda)-g_{a}\left(\theta+\lambda+\frac{\pi}{t}\right)\right|+\left|g_{a}(\theta-\lambda)-g_{a}\left(\theta-\lambda+\frac{\pi}{t}\right)\right| \leqq \frac{2 \pi}{a^{2} t} .
$$

Thus

$$
\begin{aligned}
\left|h_{1}(\theta) W(\theta)-h_{2}(\theta) W\left(\theta+\frac{\pi}{t}\right)\right| & \leqq\left|h_{1}(\theta)-h_{2}(\theta)\right| W(\theta)+\left|W(\theta)-W\left(\theta+\frac{\pi}{t}\right)\right|\left|h_{2}(\theta)\right| \\
& \leqq \frac{2 \pi}{a^{2} t} W(\theta)+\frac{2}{a}\left|W\left(\theta+\frac{\pi}{t}\right)-W(\theta)\right| .
\end{aligned}
$$

Applying these inequalities in (5.13) gives

$$
\begin{aligned}
\left|J_{2}\right| \leqq & \frac{1}{a} \int_{(B-\pi) / t}^{B / t} W\left(\theta+\frac{\pi}{t}\right) d \theta+\frac{\pi}{a^{2} t} \cdot \int_{B / t}^{A} W(\theta) d \theta \\
& +\frac{1}{a} \int_{B / t}^{A}\left|W\left(\theta+\frac{\pi}{t}\right)-W(\theta)\right| d \theta .
\end{aligned}
$$

From Lemma 3 it is clear that

$$
\lim _{t \rightarrow \infty} m(t) \int_{(B-\pi) / t}^{B / t} W\left(\theta+\frac{\pi}{t}\right) d \theta=k_{\alpha}\left[(B+\pi)^{1-\alpha}-B^{1-\alpha}\right]=O\left(\frac{1}{B^{\alpha}}\right) .
$$

Also, since $W$ is integrable on $[0, A], A<\infty$,

$$
\frac{\pi}{a^{2}} \cdot \frac{m(t)}{t} \cdot \int_{B / t}^{A} W(\theta) d \theta=O\left(\frac{m(t)}{t}\right) \rightarrow 0 \quad \text { as } t \rightarrow \infty
$$


(That $m(t) / t \rightarrow 0, t \rightarrow \infty$, follows from Lemma $1, \S 3$, in our case, but is true for any $F$ on $[0, \infty)$ with $m$ given by (1.3).) Hence from (5.14)

$$
\limsup _{t \rightarrow \infty} m(t)\left|J_{2}(t, B)\right|=a^{-1} \limsup _{t \rightarrow \infty} m(t) \int_{B / t}^{A}\left|W\left(\theta+\frac{\pi}{t}\right)-W(\theta)\right| d \theta+O\left(\frac{1}{B^{\alpha}}\right) .
$$

But $O\left(B^{-\alpha}\right)=O\left(B^{1-2 \alpha}\right)(B>1,0 \leqq \alpha \leqq 1)$, so the proof of (5.11) will be complete when we show

$$
\limsup _{t \rightarrow \infty} m(t) \int_{B / t}^{A}\left|W\left(\theta+\frac{\pi}{t}\right)-W(\theta)\right| d \theta=O\left(\frac{1}{B^{2 \alpha-1}}\right) .
$$

By Lemma 5 (i) we get

$$
\begin{aligned}
\left|W\left(\theta+\frac{\pi}{t}\right)-W(\theta)\right| & =\left|\operatorname{Re} \frac{\phi(\theta+\pi / t)-\phi(\theta)}{[1-\phi(\theta+\pi / t)][1-\phi(\theta)]}\right| \\
& \leqq \frac{2(\pi / t) m(t / \pi)}{|1-\phi(\theta+\pi / t)||1-\phi(\theta)|}
\end{aligned}
$$

Applying this estimate and the Cauchy-Schwarz inequality to the integral in (5.15) gives

$$
\begin{aligned}
\int_{B / t}^{A}\left|W\left(\theta+\frac{\pi}{t}\right)-W(\theta)\right| d \theta & \leqq \frac{2 \pi}{t} m\left(\frac{t}{\pi}\right)\left(\int_{B / t}^{A} \frac{d \theta}{1-\left.\phi(\theta+\pi / t)\right|^{2}}\right)^{1 / 2}\left(\int_{B / t}^{A} \frac{d \theta}{|1-\phi(\theta)|^{2}}\right)^{1 / 2} \\
& <8 \frac{m(t)}{t} \int_{B / t}^{2 A} \frac{d \theta}{|1-\phi(\theta)|^{2}} \quad(\pi / t \leqq A) .
\end{aligned}
$$

Again by Lemma 5(ii) there is a constant $k<\infty$ such that

for $0<\theta \leqq 2 A$. Consequently

$$
1 /|1-\phi(\theta)| \leqq k / \theta m(1 / \theta)
$$

$$
\int_{B / t}^{2 A} \frac{d \theta}{|1-\phi(\theta)|^{2}} \leqq k^{2} \int_{B / t}^{2 A} \frac{d \theta}{\theta^{2} m^{2}(1 / \theta)}=k^{2} \int_{\eta}^{t / B} \frac{d x}{m^{2}(x)}
$$

where $\eta=1 / 2 A$. Combining (5.16) and (5.17) we get

$$
\begin{aligned}
\limsup _{t \rightarrow \infty} m(t) \cdot \int_{B / t}^{A}\left|W\left(\theta+\frac{\pi}{t}\right)-W(\theta)\right| d \theta & \leqq 8 k^{2} \lim _{t \rightarrow \infty} \frac{m^{2}(t)}{t} \int_{\eta}^{t / B} \frac{d x}{m^{2}(x)} \\
& =\frac{1}{(2 \alpha-1) B^{2 \alpha-1}} \quad\left(\alpha>\frac{1}{2}\right)
\end{aligned}
$$

where the last equality comes from (3.2). This completes the proof of (5.15) and hence of (5.11).

$5^{\circ}$. The proof of (5.8) with $C=C_{\alpha}=[\Gamma(\alpha) \Gamma(2-\alpha)]^{-1}$ is now almost immediate. Let

$$
\begin{aligned}
\Delta(t) & =\left|m(t) \int_{-\infty}^{\infty} e^{-i t \theta} g_{a}(\theta+\lambda) W(\theta) d \theta-\pi C_{\alpha} g_{a}(\lambda)\right| \\
& =\left|m(t)\left(J_{1}+J_{2}\right)-\pi C_{\alpha} g_{a}(\lambda)\right|
\end{aligned}
$$


and suppose $\alpha \neq 1$. Then by (5.10) and (5.11)

$$
\begin{aligned}
\limsup _{t \rightarrow \infty} \Delta(t) & \leqq \lim _{t \rightarrow \infty}\left|m(t) J_{1}-\frac{\pi g_{a}(\lambda)}{\Gamma(\alpha) \Gamma(2-\alpha)}\right|+\limsup _{t \rightarrow \infty} m(t)\left|J_{2}\right| \\
& =\frac{g_{a}(\lambda)}{\Gamma(2-\alpha)} \cdot\left|2 \cos \left(\frac{\pi \alpha}{2}\right) \int_{0}^{B} \frac{\cos x}{x^{\alpha}} d x-\frac{\pi}{\Gamma(\alpha)}\right|+O\left(\frac{1}{B^{2 \alpha-1}}\right) .
\end{aligned}
$$

Now as $B \rightarrow \infty, \int_{0}^{B} x^{-\alpha} \cos x d x \rightarrow \sin (\pi \alpha / 2) \Gamma(1-\alpha)$, hence

$$
\lim _{B \rightarrow \infty}\left|2 \cos \left(\frac{\pi \alpha}{2}\right) \int_{0}^{B} \frac{\cos x}{x^{\alpha}} d x-\frac{\pi}{\Gamma(\alpha)}\right|=\left|\sin (\pi \alpha) \Gamma(1-\alpha)-\frac{\pi}{\Gamma(\alpha)}\right|=0 .
$$

Therefore taking the limit in (5.18) as $B \rightarrow \infty$ we get

$$
\limsup _{t \rightarrow \infty} \Delta(t)=\lim _{B \rightarrow \infty} \limsup _{t \rightarrow \infty} \Delta(t)=0
$$

which proves (5.8) when $\alpha \neq 1$. When $\alpha=1$ the proof of (5.8), with $C=C_{1}=1$, from (5.10) and (5.11) is even simpler so we omit it. Theorem 1 now follows from Lemma 8.

6. Proof of Lemma 8. There is no loss in generality in supposing $C=1$. Taking $\lambda=0$ in (5.4) and (5.6) we see that as $t \rightarrow \infty$

$$
\Delta_{t}(a)=\int_{-\infty}^{\infty} \gamma_{a}(x) \mu_{t}\{d x\} \rightarrow \int_{-\infty}^{\infty} \gamma_{a}(x) d x=\frac{2 \pi}{a}>0
$$

Hence (5.4) implies that the characteristic function of the probability measure

$$
P_{t}\{d x\}=\frac{1}{\Delta_{t}(a)} \gamma_{a}(x) \mu_{t}\{d x\}
$$

converges pointwise to the characteristic function of the probability measure

$$
P\{d x\}=(a / 2 \pi) \gamma_{a}(x) d x .
$$

Consequently, by the continuity theorem for characteristic functions $\boldsymbol{P}_{\boldsymbol{t}}$ converges weakly to $P$ as $t \rightarrow \infty$. Whence

$$
\lim _{t \rightarrow \infty} \int_{-\infty}^{\infty} B(x) \gamma_{a}(x) \mu_{t}\{d x\}=\int_{-\infty}^{\infty} B(x) \gamma_{a}(x) d x
$$

for every bounded continous function $B$ on $R^{1}$ and for every $a>0$.

For any continuous function $f$ with compact support, write

$$
\lambda_{t}(f)=\int_{-\infty}^{\infty} f(x) \mu_{t}\{d x\}, \quad \lambda(f)=\int_{-\infty}^{\infty} f(x) d x .
$$

Let $I$ be a bounded interval and let $\varepsilon>0$ be arbitrary but fixed. We can find continuous functions $f^{+}$and $f^{-}$both with compact support such that

(i) $0 \leqq f^{-} \leqq 1, f^{-}(x)=0$ for $x \notin I$,

(ii) $|I| \leqq \lambda\left(f^{-}\right)+\varepsilon$, 
(iii) $f^{+} \geqq 0, f^{+}(x)=1$ for $x$ in $I$,

(iv) $\lambda\left(f^{+}\right) \leqq|I|+\varepsilon$.

Now choose $a>0$ so small that

$$
f^{+}(x)=f^{-}(x)=0 \quad \text { when }|x| \geqq \pi / 4 a .
$$

Then since

$$
\gamma_{a}(x)=2\left(\frac{1-\cos a x}{a^{2} x^{2}}\right)>0 \text { for }|x|<\pi / 2 a
$$

it follows that $B^{+}=f^{+} / \gamma_{a}$ and $B^{-}=f^{-} / \gamma_{a}$ are continuous functions on $R^{1}$ with compact support (hence bounded). Therefore by (6.1)

$$
\lambda_{t}\left(f^{ \pm}\right)=\int_{-\infty}^{\infty} B^{ \pm}(x) \gamma_{a}(x) \mu_{t}\{d x\} \rightarrow \int_{-\infty}^{\infty} B^{ \pm}(x) \gamma_{a}(x) d x=\lambda\left(f^{ \pm}\right) .
$$

From (i) and (iii) it is clear that

$$
\lambda_{t}\left(f^{-}\right) \leqq \mu_{t}\{I\} \leqq \lambda_{t}\left(f^{+}\right)
$$

for all $t>0$. Letting $t \rightarrow \infty$ and using (6.2) we get

$$
\lambda\left(f^{-}\right) \leqq \lim \inf \mu_{t}\{I\} \leqq \lim \sup \mu_{t}\{I\} \leqq \lambda\left(f^{+}\right),
$$

and hence by (ii) and (iv)

$$
|I|-\varepsilon \leqq \lim \inf \mu_{t}\{I\} \leqq \lim \sup \mu_{t}\{I\} \leqq|I|+\varepsilon .
$$

Since this holds for every $\varepsilon>0$ it follows that

$$
\mu_{t}\{I\} \rightarrow|I|, \quad t \rightarrow \infty
$$

which completes the proof.

7. Proof of Theorem 2.

$1^{\circ}$. Our first task is to show

$$
\liminf _{t \rightarrow \infty} m(t)(U(t+h)-U(t)) \geqq C_{\alpha} h \quad(h>0),
$$

or, equivalently,

$$
\liminf _{t \rightarrow \infty} t^{1-\alpha} L(t)(U(t+h)-U(t)) \geqq \frac{\sin \pi \alpha}{\pi} h .
$$

(See remark following the statement of Theorem 2.)

Condition (1.2) with $0<\alpha<1$ is necessary and sufficient for $F$ to be in the domain of attraction of the unique (apart from a scale factor) stable distribution with exponent $\alpha$ concentrated on $[0, \infty)$. Thus if a sequence $\left\{B_{n}\right\}$ is chosen so that $0<B_{n} \uparrow \infty$ and

as $n \rightarrow \infty$, then

$$
n\left(1-F\left(B_{n}\right)\right) \equiv n B_{n}^{-\alpha} L\left(B_{n}\right) \rightarrow 1
$$

$$
F^{n^{*}}\left(B_{n} x\right) \rightarrow \int_{0}^{x} q_{a}(y) d y \quad(n \rightarrow \infty, x \geqq 0)
$$


where $q_{\alpha}>0$ and satisfies

$$
\int_{0}^{\infty} e^{-\lambda y} q_{\alpha}(y) d y=\exp \left[-\lambda^{\alpha} \Gamma(1-\alpha)\right], \quad \lambda \geqq 0 .
$$

In addition to (7.3) a local limit theorem for nonarithmetic distributions due to C. Stone [9] implies the somewhat stronger result

$$
F^{k^{*}}(t+h)-F^{k^{*}}(t)=\left(h / B_{k}\right) q_{\alpha}\left(t / B_{k}\right)+\delta_{k} / B_{k}
$$

where $\delta_{k} \rightarrow 0$ as $k \rightarrow \infty$ uniformly in $t>0\left((7.3)\right.$ only allows $F^{k^{*}}(t+h)-F^{k^{*}}(t)$ $\sim h B_{k}^{-1} q_{\alpha}\left(t B_{k}^{-1}\right)$ for $t$ and $h$ fixed). Using (7.4) we prove (7.2) almost exactly as Garsia and Lamperti [5] prove the analogous inequality in the arithmetic case. Thus from (1.1) and (7.4)

$$
\begin{aligned}
U(t+h)-U(t) & >\sum_{k=n}^{r}\left(F^{k^{*}}(t+h)-F^{k^{*}}(t)\right) \\
& =h \sum_{n}^{r} \frac{1}{B_{k}} q_{\alpha}\left(\frac{t}{B_{k}}\right)+\sum_{n} \frac{\delta_{k}}{B_{k}}
\end{aligned}
$$

Let $0<A<C<\infty$, and choose $n=\left[A t^{\alpha} / L(t)\right], r=\left[C t^{\alpha} / L(t)\right]$. Then, as in [5], we have both

$$
t^{1-\alpha} L(t) \sum_{n}^{r} \frac{\delta_{k}}{B_{k}}=o(1), \quad t \rightarrow \infty
$$

and, writing $x_{k}=k L(t) / t^{\alpha}, n \leqq k \leqq r$,

as $t \rightarrow \infty$. Hence for any $\varepsilon>0$

$$
\begin{aligned}
t^{1-\alpha} L(t) \sum_{n}^{r} \frac{1}{B_{k}} q_{\alpha}\left(\frac{t}{B_{k}}\right) & \sim \sum_{A \leqq x_{k} \leqq C} x_{k}^{-1 / \alpha} q_{\alpha}\left(x_{k}^{-1 / \alpha}\right)\left(x_{k+1}-x_{k}\right) \\
& \rightarrow \int_{A}^{C} x^{-1 / \alpha} q_{\alpha}\left(x^{-1 / \alpha}\right) d x
\end{aligned}
$$

$$
t^{1-\alpha} L(t)(U(t+h)-U(t)) \geqq \int_{A}^{C} x^{-1 / \alpha} q_{\alpha}\left(x^{-1 / \alpha}\right) d x-\varepsilon
$$

for all $t$ sufficiently large. In other words

$$
\liminf _{t \rightarrow \infty} t^{1-\alpha} L(t)(U(t+h)-U(t)) \geqq \int_{A}^{C} x^{-1 / \alpha} q_{\alpha}\left(x^{-1 / \alpha}\right) d x,
$$

and (7.2) now follows by letting $A \rightarrow 0, C \rightarrow \infty$ and noting

$$
\int_{0}^{\infty} x^{-1 / \alpha} q_{\alpha}\left(x^{-1 / \alpha}\right) d x=\alpha \int_{0}^{\infty} y^{-\alpha} q_{\alpha}(y) d y=\frac{\sin \pi \alpha}{\pi} .
$$

$2^{\circ}$. To complete the proof of Theorem 2 we need the following lemma (also needed in the proof of Theorem 3).

LEMMA 9. Let $z$ be any nonnegative integrable (but not necessarily dri) function on $[0, \infty)$. Then

$$
\liminf _{t \rightarrow \infty} m(t) \int_{0}^{t} z(t-y) U\{d y\} \leqq C_{\alpha} \int_{0}^{\infty} z(x) d x \quad(0<\alpha \leqq 1) .
$$


To finish the proof of Theorem 2 we set $z(x)=1$ for $0 \leqq x \leqq h, z(x)=0$ elsewhere. Noting that $m(t+h) \sim m(t)$ as $t \rightarrow \infty$ we get from (7.5)

$$
\begin{aligned}
\liminf _{t \rightarrow \infty} m(t)(U(t+h)-U(t)) & =\liminf _{t \rightarrow \infty} m(t+h) U^{*} z(t+h) \\
& \leqq C_{\alpha} \int_{0}^{\infty} z(x) d x=C_{\alpha} h .
\end{aligned}
$$

Together (7.1) and (7.6) give (1.5).

Proof of Lemma 9. Let $v(t)=U^{*} z(t)=\int_{0}^{t} z(t-x) U\{d x\}$. Then

$$
\hat{v}(\lambda)=\int_{0}^{\infty} e^{-\lambda x} v(x) d x=\left(\int_{0}^{\infty} e^{-\lambda x} z(x) d x\right) \hat{U}(\lambda)=\hat{z}(\lambda) \hat{U}(\lambda)
$$

where $U$ is defined as in $\S 2(i)$. Since $U$ is regularly varying with exponent $\alpha$ we have

$$
\hat{U}(\lambda) \sim \Gamma(\alpha+1) U(1 / \lambda) \text { as } \lambda \rightarrow 0+
$$

by Theorem 1 in $\left[3\right.$, p. 420]. Now $\hat{z}(0)=\int_{0}^{\infty} z(x) d x<\infty$ and it follows that

$$
\hat{v}(\lambda) \sim \hat{z}(0) \Gamma(\alpha+1) U(1 / \lambda), \quad \lambda \rightarrow 0+
$$

which, by the converse of the same Theorem 1 in [3], is the same as

$$
\int_{0}^{t} v(x) d x \sim \hat{z}(0) U(t), \quad t \rightarrow \infty
$$

Now by Theorem 5 in $\S 2$

$$
U(t) \sim(\Gamma(\alpha+1) \Gamma(2-\alpha))^{-1} t / m(t)=\left(C_{\alpha} / \alpha\right) t / m(t)
$$

as $t \rightarrow \infty$; also, since $1 / m$ is regularly varying with exponent $\alpha-1>-1$ we have for fixed $\eta>0$

$$
\frac{1}{\alpha} \frac{t}{m(t)} \sim \int_{\eta}^{t} \frac{d x}{m(x)}, \quad t \rightarrow \infty
$$

(cf. [3, p. 273]). From (7.7), (7.8), and (7.9) it follows that

$$
\int_{0}^{t} v(x) d x \sim C_{\alpha} \hat{z}(0) \int_{\eta}^{t} \frac{d x}{m(x)}, \quad t \rightarrow \infty .
$$

Suppose, contrary to (7.5),

$$
\liminf _{t \rightarrow \infty} m(t) v(t)>C_{\alpha} \hat{z}(0) .
$$

Then for some $\varepsilon>0$ and all $x \geqq \eta$ sufficiently large

$$
v(x) \geqq(1+\varepsilon) C_{\alpha} \hat{z}(0)(1 / m(x)) .
$$

Hence

$$
\int_{0}^{t} v(x) d x \geqq \int_{\eta}^{t} v(x) d x \geqq(1+\varepsilon) C_{\alpha} \hat{z}(0) \int_{\eta}^{t} \frac{d x}{m(x)}
$$

for all $t \geqq \eta$. But this contradicts (7.10). 
8. Proof of Theorems 3 and 4.

$1^{\circ}$. Let $h>0$. Throughout this section put $z_{k}(x)=1$ when $(k-1) h \leqq x<k h$, $z_{k}(x)=0$ elsewhere, and let

$$
v_{k}(t)=U^{*} z_{k}(t)=U(t-(k-1) h)-U(t-k h) .
$$

Since $m(t-k h) \sim m(t)$ for fixed $k h, t \rightarrow \infty$, we have by Theorems 1 and 2

$$
\begin{aligned}
& \liminf _{t \rightarrow \infty} m(t) v_{k}(t)=C_{\alpha} h\left(0<\alpha \leqq \frac{1}{2}\right), \\
& \lim _{t \rightarrow \infty} m(t) v_{k}(t)=C_{\alpha} h \quad\left(\frac{1}{2}<\alpha \leqq 1\right) ; k=1,2, \ldots
\end{aligned}
$$

$2^{\circ}$. Let $z \geqq 0$ be any dri function on $[0, \infty]$. Then

$$
\liminf _{t \rightarrow \infty} m(t) \int_{0}^{t} z(t-y) U\{d y\} \geqq C_{\alpha} \int_{0}^{\infty} z(x) d x \quad(0<\alpha \leqq 1) .
$$

Theorem 4 follows immediately from (8.2) and Lemma 9.

To prove (8.2) let $\varepsilon>0$ be arbitrary. We suppose $h>0$ is so small that

$$
\int_{0}^{\infty} z(x) d x-\frac{\varepsilon}{C_{\alpha}}<\sum_{1}^{\infty} a_{k} h
$$

where $a_{k}=\inf \{z(x):(k-1) h \leqq x<k h\}$. Then by (8.1) and Fatou's lemma

$$
\begin{aligned}
C_{\alpha} \int_{0}^{\infty} z(x) d x-\varepsilon & <\sum_{1}^{\infty} a_{k} \liminf _{t \rightarrow \infty} m(t) v_{k}(t) \\
& \leqq \liminf _{t \rightarrow \infty} m(t) \sum_{1}^{\infty} a_{k} U^{*} z_{k}(t) \\
& \leqq \liminf _{t \rightarrow \infty} m(t) U^{*} z(t)
\end{aligned}
$$

which implies (8.2) as $\varepsilon>0$ is arbitrary.

$3^{\circ}$. From now on in addition to being dri we assume $z$ satisfies (1.7). That is for some constant $b<\infty$

$$
0 \leqq z(x) \leqq b / x, \quad x>0 .
$$

We also assume $\frac{1}{2}<\alpha \leqq 1$ in (1.2). Obviously our goal now is to show

$$
\limsup _{t \rightarrow \infty} m(t) \int_{0}^{t} z(t-y) U\{d y\} \leqq C_{\alpha} \int_{0}^{\infty} z(x) d x .
$$

$4^{\circ}$. Fix $0<\theta<1$. Then

$$
\limsup _{t \rightarrow \infty} m(t) \int_{0}^{t \theta} z(t-y) U\{d y\} \leqq \frac{b C_{\alpha} \theta^{\alpha}}{\alpha(1-\theta)}
$$

and

$$
\limsup _{t \rightarrow \infty} m(t) \int_{t \theta}^{t} z(t-y) U\{d y\} \leqq C_{\alpha} \int_{0}^{\infty} z(x) d x
$$


Proof of (8.5). From (8.3)

$$
\int_{0}^{t \theta} z(t-y) U\{d y\} \leqq b \int_{0}^{t \theta} \frac{1}{t-y} U\{d y\} \leqq \frac{b}{(1-\theta) t} U(t \theta) .
$$

But $U(t \theta) \sim \theta^{\alpha} U(t) \sim \alpha^{-1} C_{\alpha} \theta^{\alpha}(t / m(t))$ as $t \rightarrow \infty$ by Theorem 5 and Lemma 1 . Hence

$$
\limsup _{t \rightarrow \infty} m(t) \int_{0}^{t \theta} z(t-y) U\{d y\} \leqq \frac{b}{1-\theta} \lim _{t \rightarrow \infty} \frac{m(t)}{t} U(t \theta)=\frac{b C_{\alpha} \theta^{\alpha}}{\alpha(1-\theta)} .
$$

Proof of (8.6). Let $\varepsilon>0$ be arbitrary and put $b_{k}=\sup \{z(x):(k-1) h \leqq x<k h\}$. We assume $h$ is so small that

$$
\sum_{1}^{\infty} b_{k} h<\int_{0}^{\infty} z(x) d x+\frac{\varepsilon}{C_{\alpha}}
$$

Let $n$ be the largest integer satisfying $(n-1) h \leqq t(1-\theta)$. Then $z_{k}(t-y)=0$ for $k \geqq n+1$ and all $t \theta \leqq y \leqq t$, hence

$$
\int_{t \theta}^{t} z(t-y) U\{d y\} \leqq \sum_{1}^{n} b_{k} \int_{t \theta}^{t} z_{k}(t-y) U\{d y\} \leqq \sum_{1}^{n} b_{k} v_{k}(t)
$$

Suppose for the moment that

$$
\lim _{t \rightarrow \infty} m(t) \sum_{1}^{n} b_{k} v_{k}(t)=C_{\alpha} \sum_{1}^{\infty} b_{k} h
$$

Then by (8.8) and (8.7)

$$
\limsup _{t \rightarrow \infty} m(t) \int_{t \theta}^{t} z(t-y) U\{d y\} \leqq C_{\alpha} \sum_{1}^{\infty} b_{k} h<C_{\alpha} \int_{0}^{\infty} z(x) d x+\varepsilon
$$

which yields (8.6) on letting $\varepsilon \rightarrow 0$.

Let $\beta_{t}(k)=b_{k} m(t) v_{k}(t)$ for $k=1,2, \ldots, n$ and $\beta_{t}(k)=0$ for $k \geqq n+1$ then $m(t) \sum_{1}^{n} b_{k} v_{k}(t)=\sum_{k=1}^{\infty} \beta_{t}(k)$, and since, by $(8.1), \beta_{t}(k) \rightarrow C_{\alpha} h b_{k}, k=1,2, \ldots, t \rightarrow \infty$, we see that to establish (8.9) it will suffice to find numbers $T$ and $B$ so that

$$
\beta_{t}(k) \leqq B b_{k} \quad \text { for all } k \geqq 1 \text { and all } t \geqq T \text {. }
$$

First choose $s_{0}$ so that $s \geqq s_{0}$ implies

$$
U(s+h)-U(s)<2 C_{\alpha} h / m(s) .
$$

Next from $m(t \theta-h) \sim m(t \theta) \sim \theta^{1-\alpha} m(t)$ as $t \rightarrow \infty$, we find a $t_{0}$ so that for all $t \geqq t_{0}$

$$
m(t)<2 \theta^{\alpha-1} m(t \theta-h) .
$$

Suppose now that $t \geqq t_{0}, t \theta-h \geqq s_{0}$ and $1 \leqq k \leqq n$. Noting that $t \theta-h \leqq t-k h$, by definition of $n$, we get

and

$$
m(t)<2 \theta^{\alpha-1} m(t \theta-h) \leqq 2 \theta^{\alpha-1} m(t-k h)
$$

$$
v_{k}(t)=U(t-k h+h)-U(t-k h)<2 C_{\alpha} h / m(t-k h),
$$


that is, $m(t) v_{k}(t)<4 C_{\alpha} h \theta^{\alpha-1}$. Since $\beta_{t}(k)=0$ for $k>n$ we see that (8.10) holds with $T=\max \left\{\left(s_{0}+h\right) / \theta, t_{0}\right\}$ and $B=4 C_{\alpha} h \theta^{\alpha-1}$. This completes the proof of (8.6).

$5^{\circ}$. From (8.5) and (8.6) we have

$$
\begin{aligned}
\limsup _{t \rightarrow \infty} m(t) U^{*} z(t) & =\limsup _{t \rightarrow \infty} m(t)\left(\int_{0}^{t \theta}+\int_{t \theta}^{t}\right) z(t-y) U\{d y\} \\
& =O\left(\frac{\theta^{\alpha}}{1-\theta}\right)+C_{\alpha} \int_{0}^{\infty} z(x) d x
\end{aligned}
$$

whenever $0<\theta<1$. Letting $\theta \rightarrow 0$ gives (8.4).

Theorem 3 is evident from (8.2) and (8.4).

9. An application. In this section we study the asymptotic behavior of the spent and residual waiting times associated with a renewal process whose waiting time distribution has the form (1.2) with $\alpha=1$.

A renewal process with waiting time distribution $F$ is any sequence $\left\{S_{n}\right\}, n \geqq 0$ of the form $S_{0}=0, S_{n}=X_{1}+\cdots+X_{n}, n \geqq 1$, where the $X_{n}$ are positive mutually independent random variables with common distribution $F$. The $S_{n}$ are usually interpreted as consecutive points on a time axis and are called renewal epochs. The $X_{n}$ are then called waiting times. In this context $U\{I\}=\sum F^{n^{*}}\{I\}=\sum P\left\{S_{n} \in I\right\}$ is clearly the expected number of renewal epochs falling in $I$.

Our interest here is in two auxiliary random variables $Y_{t}$ and $Z_{t}$ called, respectively, the spent and residual (or excess) waiting time at epoch $t$ defined as follows: let $N_{t}=\max \left\{n: S_{n} \leqq t\right\}$ (=the number of renewal epochs in $\left.(0, t]\right)$. Then

$$
Y_{t}=t-S_{N_{t}}, \quad Z_{t}=S_{N_{t}+1}-t .
$$

When the distribution $F$ has a finite mean, $Y_{t}$ and $Z_{t}$ have nondegenerate limit distributions:

$$
\lim _{t \rightarrow \infty} P\left\{Y_{t}>y, Z_{t}>z\right\}=\frac{1}{\mu} \int_{y+z}^{\infty}[1-F(u)] d u
$$

(see [3, p. 371, problem 3], or [2, Theorem 1]).

In general when $\mu=\infty$ the most one can say is $Y_{t} \rightarrow \infty$ and $Z_{t} \rightarrow \infty$ in probability. However, if $F$ has the form (1.2) with $0<\alpha<1$, then Lamperti [7] and Dynkin [2] have shown that $Y_{t} / t$ and $Z_{t} / t$ have nontrivial limit distributions:

$$
\lim _{t \rightarrow \infty} P\left\{\frac{Y_{t}}{t}>y, \frac{Z_{t}}{t}>z\right\}=\frac{\sin \pi \alpha}{\pi} \int_{y}^{1}(z+u)^{-\alpha}(1-u)^{\alpha-1} d u,
$$

for $0 \leqq z<\infty$ and $0 \leqq y \leqq 1$. See also Feller [3, p. 447]. These writers show that (1.2) with $0<\alpha<1$ is in fact necessary and sufficient for $Y_{t} / t$ and $Z_{t} / t$ to have nontrivial limit distributions. (Dynkin proves that if $Y_{t} / \beta(t)$ (or $Z_{t} / \beta(t)$ ) has a nontrivial limit distribution where $\beta(t)$ is regularly varying and approaches infinity as $t \rightarrow \infty$, then (1.2) holds for some $0<\alpha<1$ and $\beta(t) / t \rightarrow$ const.)

When $\alpha=1$ in (1.2) $F$ may or may not have a finite mean (see $\S 2(v)$ ), but in either case it is quite straightforward to show that $Y_{t} / t \rightarrow 0$ and $Z_{t} / t \rightarrow 0$ in probability 
(see (9.4) for the precise rate). But as noted above if $\mu=\infty$ we also have $Y_{t}$ and $Z_{t} \rightarrow \infty$ (in probability) so one might expect that some nonlinear normalization such as $\lambda\left(Y_{t}\right) / \beta(t)$ where $\lambda(t), \beta(t) \rightarrow \infty$ will yet produce a nontrivial limit distribution.

THEOREM 6. Let $F$ have the form

$$
1-F(t)=L(t) / t, \quad t>0,
$$

where $L$ is slowly varying at $\infty$ and suppose the mean of $F$ is infinite. Then for $0 \leqq a \leqq 1, b \geqq 0$

$$
\lim _{t \rightarrow \infty} P\left\{\frac{m\left(Y_{t}\right)}{m(t)} \leqq a, \frac{m\left(Z_{t}\right)}{m(t)} \leqq b\right\}=\min \{a, b\}
$$

where $m$ is the function defined by (1.3).

The limit distribution in (9.2) is just the uniform distribution concentrated on the diagonal of the unit square, consequently we have the following.

Corollary. $\left(m\left(Y_{t}\right)-m\left(Z_{t}\right)\right) / m(t) \rightarrow 0$ in probability as $t \rightarrow \infty$, and for $0<\theta<1$

$$
\lim _{t \rightarrow \infty} P\left\{\frac{m\left(Y_{t}\right)}{m(t)} \leqq \theta\right\}=\lim _{t \rightarrow \infty} P\left\{\frac{m\left(Z_{t}\right)}{m(t)} \leqq \theta\right\}=\theta .
$$

Remarks. 1. Since $Z_{t}$ and $Y_{t} \rightarrow \infty$ in probability it is clear that the function $m$ in these results may be replaced by any function $m_{1}$ such that $m_{1}(t) \uparrow \infty$ and $m_{1}(t) / m(t) \rightarrow k \neq 0$ as $t \rightarrow \infty$.

2. It should be pointed out that for any $F$ on $(0, \infty)$ with a finite mean $(9.3)$ (but not (9.2)) is still valid. To see this consider for example $Y_{t}$. Let $\rho$ be the continuous inverse of $m: \rho(m(t))=t, m(\rho(x))=x, 0 \leqq x<\mu$. From (9.1),

hence

$$
\lim _{t \rightarrow \infty} P\left\{Y_{t} \leqq y\right\}=\mu^{-1} \int_{0}^{y}[1-F(x)] d x=m(y) / \mu
$$

$\lim _{t \rightarrow \infty} P\left\{m\left(Y_{t}\right) / m(t) \leqq \theta\right\}=\lim _{t \rightarrow \infty} P\left\{Y_{t} \leqq \rho(\theta \mu)\right\}=m(\rho(\theta \mu)) / \mu=\theta \quad(0<\theta<1)$.

Our last result gives precise information about the distribution of $Y_{t} / t$ and $Z_{t} / t$ for large $t$.

THEOREM 7. Let $F$ be as in Theorem 6 and let $0 \leqq a \leqq 1, b \geqq 0, a+b \neq 0$. Then as $t \rightarrow \infty$

$$
P\left\{\frac{Y_{t}}{t}>a, \frac{Z_{t}}{t}>b\right\} \sim \frac{L(t)}{m(t)} \cdot \log \left(\frac{1+b}{a+b}\right) .
$$

(Note that $L(t) / m(t) \rightarrow 0$ as $t \rightarrow \infty$ by Lemma 1.)

Proof. From (9.7) it follows that

$$
\begin{aligned}
G_{t}(a, b) & =P\left\{Y_{t}>t a, Z_{t}>t b\right\}=\int_{0}^{t-a t}[1-F(t+t b-x)] U\{d x\} \\
& =\int_{0}^{1-a}[1-F(t(1+b-y))] U\{t d y\} .
\end{aligned}
$$


We now argue as in the proof of (2.8): By Lemma 1 and Theorem 5 (with $\alpha=1$ )

so

$$
[1-F(t)] U(t) \sim L(t) / m(t), \quad t \rightarrow \infty,
$$

Now

$$
G_{t}(a, b) \frac{m(t)}{L(t)} \sim \int_{0}^{1-a} \frac{1-F(t(1+b-y))}{1-F(t)} \cdot \frac{U\{t d y\}}{U(t)}, \quad t \rightarrow \infty .
$$

$$
f_{t}(y)=\frac{1-F(t(1+b-y))}{1-F(t)} \rightarrow \frac{1}{1+b-y} \text { as } t \rightarrow \infty
$$

and the convergence is uniform in $0 \leqq y \leqq 1-a$ (provided $a+b \neq 0$ ) since each $f_{t}(y)$ is monotone in $y$ and since the limit $1 /(1+b-y)$ is continuous on $0 \leqq y \leqq 1-a$. Also, since $U(t y) / U(t) \rightarrow y$, the measure $U\{t d y\} / U(t)$ converges weakly to Lebesgue measure as $t \rightarrow \infty$.

From these remarks we see that

$$
P\left\{Y_{t}>t a, Z_{t}>t b\right\} \frac{m(t)}{L(t)} \rightarrow \int_{0}^{1-a} \frac{1}{1+b-y} d y, \quad t \rightarrow \infty,
$$

and (9.4) follows.

Proof of Theorem 6. Since we use Theorem 1 we shall assume $F$ is nonarithmetic. Theorem 6 is still true when $F$ is arithmetic, and, though certain of the details in the present proof must be slightly modified, the essential points are the same. (Of course one uses (2.4) rather than Theorem 1 in the arithmetic case.)

Let $\rho$ be the strictly increasing continuous inverse of the function $m: \rho(m(t))$ $=m(\rho(t))=t$. Since $F$ has infinite expectation, $m(t) \rightarrow \infty$ as $t \rightarrow \infty$ so $\rho$ is defined on $[0, \infty)$. Fix $0<a<1, b>0$ and let

$$
a_{t}=\rho(a m(t)), \quad b_{t}=\rho(b m(t)) .
$$

We will prove

$$
\lim _{t \rightarrow \infty} P\left\{Y_{t} \leqq a_{t}, Z_{t}>b_{t}\right\}=\max \{a, b\}-b
$$

which is evidently the same as (9.2).

Our starting point in proving (9.6) is the following equation

$$
P\left\{Y_{t} \leqq a, Z_{t}>b\right\}=\int_{t-a}^{t}[1-F(t+b-y)] U\{d y\} .
$$

Here is a probabilistic derivation: By definition $Y_{t}=t-S_{N_{t}}, Z_{t}=S_{N_{t}+1}-t$ where $N_{t}=n$ if and only if $S_{n} \leqq t<S_{n+1}$. Hence the joint event $\left\{Y_{t} \leqq a, Z_{t}>b\right\}$ occurs if and only if for some (unique) $n, S_{n}=y$ with $t-a \leqq y \leqq t$ and then $Z_{t}=S_{n+1}-t$ $=X_{n+1}+y-t>b$. By independence of $S_{n}$ and $X_{n+1}$, the conditional probability of the second event is simply $P\left\{X_{n+1}>t+b-y\right\}=1-F(t+b-y)$. Multiplying this by $F^{n^{*}}\{d y\}$, the distribution of $S_{n}$, and summing over all $t-a \leqq y \leqq t$ we get

$$
P\left\{Y_{t} \leqq a, Z_{t}>b, N_{t}=n\right\}=\int_{t-a}^{t}[1-F(t+b-y)] F^{n^{*}}\{d y\}
$$


Summing over all $n \geqq 0$ gives (9.7) since $\sum F^{n^{*}}=U$.

LemMA 10. (i) Let $a_{t}$ be defined by (9.5) with $0<a<1$. Then

$$
a_{t} / t \rightarrow 0 \text { but } a_{t} \rightarrow \infty \text { as } t \rightarrow \infty \text {. }
$$

(ii) Let $\varepsilon, \delta>0$. Then there is a $T>0$ such that for all $t \geqq T$ and all $\frac{1}{2} t \leqq y \leqq 2 t$ we have

$$
\frac{1-\varepsilon}{m(t)} \delta<U(y+\delta)-U(y)<\frac{1+\varepsilon}{m(t)} \delta .
$$

(We prove Lemma 10 later.)

Let $\varepsilon, \delta>0$ with $0<\varepsilon<1$ be fixed but arbitrary. By Lemma 10, $a_{t} \rightarrow \infty$ and $\left(t-a_{t}\right) / t \rightarrow 1$ as $t \rightarrow \infty$. Hence by choosing $T_{1}$ sufficiently large we may assume that both (9.9) and the inequalities

$$
\frac{1}{2} t+10 \delta<t-a_{t}<t<2 t-10 \delta, \quad a_{t}>100 \delta,
$$

hold simultaneously for all $t \geqq T_{1}$. Let $t \geqq T_{1}$ and consider the partition $0=y_{0}$ $<y_{1}<y_{2}<\cdots$ of $[0, \infty)$ where $y_{k}=k \delta$. Write

$$
\Delta U_{k}=U\left(y_{k+1}\right)-U\left(y_{k}\right)=U\left(y_{k}+\delta\right)-U\left(y_{k}\right)
$$

and let $y_{r}$ and $y_{n}$ be chosen as in the following diagram

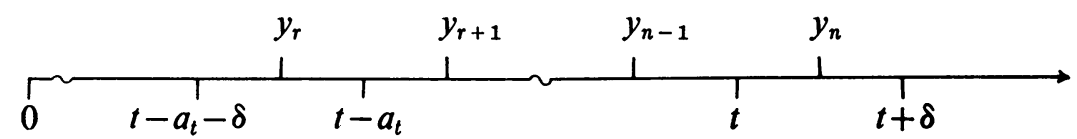

$\left(y_{r} \leqq t-a_{t}, y_{n-1} \leqq t\right)$. Since $y_{r}>t-a_{t}-\delta$ and $y_{n}<t+\delta$ it follows from (9.9) and (9.10) that

$$
\frac{1-\varepsilon}{m(t)} \delta<\Delta U_{k}<\frac{1+\varepsilon}{m(t)} \delta, \quad k=r, r+1, \ldots, n-1, n .
$$

Now let $f(y)=1-F\left(t+b_{t}-y\right), 0 \leqq y \leqq t+b_{t}$. Then $f$ is nonnegative, nondecreasing and bounded by 1 . Consequently by (9.7), (9.11) and (9.12)

$$
\begin{aligned}
P\left\{Y_{t} \leqq a_{t}, Z_{t}>b_{t}\right\} & =\int_{t-a_{t}}^{t} f(y) U\{d y\} \leqq \sum_{k=r}^{n-1} f\left(y_{k+1}\right) \Delta U_{k}<\frac{1+\varepsilon}{m(t)} \sum_{k=r}^{n-1} f\left(y_{k+1}\right) \delta \\
& =\frac{1+\varepsilon}{m(t)} \sum_{k=r+1}^{n} f\left(y_{k}\right) \delta \leqq \frac{1+\varepsilon}{m(t)} \int_{y_{r+1}}^{y_{n+1}} f(y) d y \\
& \leqq \frac{1+\varepsilon}{m(t)} \int_{t-a_{t}}^{t+2 \delta} f(y) d y \leqq \frac{1+\varepsilon}{m(t)} \int_{t-a_{t}}^{t} f(y) d y+\frac{4 \delta}{m(t)}
\end{aligned}
$$

A similar calculation gives

$$
P\left\{Y_{t} \leqq a_{t}, Z_{t}>b_{t}\right\}>\frac{1-\varepsilon}{m(t)} \int_{t-a_{t}}^{t} f(y) d y-\frac{4 \delta}{m(t)}
$$


But

$$
\begin{aligned}
\int_{t-a_{t}}^{t} f(y) d y & =\int_{t-a_{t}}^{t}\left[1-F\left(t+b_{t}-y\right)\right] d y=m\left(a_{t}+b_{t}\right)-m\left(b_{t}\right) \\
& =m\left(a_{t}+b_{t}\right)-b m(t) .
\end{aligned}
$$

Therefore for all $t \geqq T_{1}$

$$
P\left\{Y_{t} \leqq a_{t}, Z_{t}>b_{t}\right\} \lessgtr(1 \pm \varepsilon)\left(\left(\frac{m\left(a_{t}+b_{t}\right)}{m(t)}\right)-b\right) \pm \frac{4 \delta}{m(t)}
$$

Assume for the moment

$$
\lim _{t \rightarrow \infty} \frac{m\left(a_{t}+b_{t}\right)}{m(t)}=\max \{a, b\} .
$$

Then since $m(t) \rightarrow \infty$ as $t \rightarrow \infty$ we conclude from (9.13) and (9.14):

$$
\begin{aligned}
(1-\varepsilon)(\max \{a, b\}-b) & \leqq \lim \inf P\left\{Y_{t} \leqq a_{t}, Z_{t}>b\right\} \\
& \leqq \lim \sup P\left\{Y_{t} \leqq a_{t}, Z_{t}>b\right\} \\
& \leqq(1+\varepsilon)(\max \{a, b\}-b)
\end{aligned}
$$

and (9.6) follows.

It remains to prove (9.14). Let $c=\max \{a, b\}$ and $c_{t}=\rho(c m(t))$. Then $c m(t)$ $=m\left(c_{t}\right) \leqq m\left(a_{t}+b_{t}\right) \leqq m\left(2 c_{t}\right)$, or

$$
c \leqq m\left(a_{t}+b_{t}\right) / m(t) \leqq m\left(2 c_{t}\right) / m(t)=\left(m\left(2 c_{t}\right) / m\left(c_{t}\right)\right) c .
$$

Now $m$ is slowly varying by Lemma 1 and $c_{t} \rightarrow \infty$ by Lemma 10, hence

$$
m\left(2 c_{t}\right) / m\left(c_{t}\right) \rightarrow 1
$$

as $t \rightarrow \infty$. Letting $t \rightarrow \infty$ in (9.15) gives (9.14). This completes the proof of Theorem 6.

Proof of Lemma 10. (i) Since both $\rho(t) \rightarrow \infty$ and $m(t) \rightarrow \infty$ it is clear that $a_{t}=\rho(a m(t)) \rightarrow \infty$ as $t \rightarrow \infty$ for any $a>0$. Let $0<a<b$ we show

$$
\rho(\operatorname{am}(t)) / \rho(b m(t))=a_{t} / b_{t} \rightarrow 0, \quad t \rightarrow \infty .
$$

To get (9.8) take $b=1,0<a<1$ in (9.16).

Suppose (9.16) fails. Then for some $0<\theta<1$ and some sequence $t_{n} \rightarrow \infty$ we have $\theta \leqq a_{t_{n}} / b_{t_{n}} \leqq 1$ for all $n$. Hence $m\left(\theta b_{t_{n}}\right) \leqq m\left(a_{t_{n}}\right)<m\left(b_{t_{n}}\right)$, or since $m\left(a_{t}\right)=a m(t)$, $m\left(b_{t}\right)=b m(t)$,

$$
m\left(\theta b_{t_{n}}\right) / m\left(b_{t_{n}}\right) \leqq a / b<1 .
$$

But $m\left(\theta b_{t_{n}}\right) / m\left(b_{t_{n}}\right) \rightarrow 1$ as $t_{n} \rightarrow \infty$, since $m$ is slowly varying and $b_{t_{n}} \rightarrow \infty$, so (9.17) leads to the contradiction $1 \leqq a / b<1$. Hence (9.16) must be true.

(ii) Let $\varepsilon, \varepsilon_{1}, \varepsilon_{2}, \delta$ be positive numbers with $\varepsilon_{1}, \varepsilon_{2}<1$. Since $m$ is slowly varying there is a $t_{1}>0$ such that

$$
1-\varepsilon_{1}<m(t / 2) / m(2 t)<1+\varepsilon_{1} \text { for all } t \geqq t_{1} .
$$


By Theorem 1, $\alpha=1$, we can find $t_{2}>0$ so that

$$
\left(1-\varepsilon_{2}\right) \cdot \frac{\delta}{m(y)}<U(y+\delta)-U(y)<\left(1+\varepsilon_{2}\right) \cdot \frac{\delta}{m(y)}, \text { for } y \geqq t_{2} .
$$

Suppose now that $\frac{1}{2} t \geqq \max \left\{t_{1}, t_{2}\right\}$ and $\frac{1}{2} t \leqq y \leqq 2 t$. Then since $m$ is increasing

$$
m(t / 2) / m(2 t) \leqq m(t) / m(y) \leqq m(2 t) / m(t / 2) .
$$

Consequently $1-\varepsilon_{1}<m(t) / m(y)<1 /\left(1-\varepsilon_{1}\right)$ by (9.18), and from (9.19) it follows that

$$
\left(1-\varepsilon_{1}\right)\left(1-\varepsilon_{2}\right) \frac{\delta}{m(t)}<U(y+\delta)-U(y)<\left(\frac{1+\varepsilon_{2}}{1-\varepsilon_{1}}\right) \frac{\delta}{m(t)} .
$$

By (pre) choosing $\varepsilon_{1}, \varepsilon_{2}$ so that $\left(1-\varepsilon_{1}\right)\left(1-\varepsilon_{2}\right) \geqq 1-\varepsilon$ and $\left(1+\varepsilon_{2}\right) /\left(1-\varepsilon_{1}\right) \leqq 1+\varepsilon$ we get (9.9) with $T=\max \left\{2 t_{1}, 2 t_{2}\right\}$.

\section{BIBLIOGRAPHY}

1. L. Breiman, Probability, Addison-Wesley, Reading, Mass., 1968. MR 37 \#4841.

2. E. B. Dynkin, Some limit theorems for sums of independent random variables with infinite mathematical expectations, Izv. Akad. Nauk SSSR Ser. Mat. 19 (1955), 247-266; English transl., Selected Transl. Math. Stat. and Prob., vol. 1, Amer. Math. Soc., Providence, R. I., 1961, pp. 171-189. MR 17, 865; MR 22 \#7164.

3. W. Feller, Introduction to probability theory and its applications. Vol. II, Wiley, New York, 1966. MR 35 \#1048.

4. W. Feller and S. Orey, A renewal theorem, J. Math. Mech. 10 (1961), 619-624. MR 24 \#A581.

5. A. Garsia and J. Lamperti, $A$ discrete renewal theorem with infinite mean, Comment. Math. Helv. 37 (1962/63), 221-234. MR 26 \#5630.

6. G. H. Hardy and W. W. Rogosinski, Notes on Fourier series. III: Asymptotic formulae for the sums of certain trigonometrical series, Quart. J. Math. Oxford Ser. 16 (1945), 49-58. MR 7, 247.

7. J. Lamperti, Some limit theorems for stochastic processes, J. Math. Mech. 7 (1958), 433-448. MR 20 \#4888.

8. F. L. Spitzer, Principles of random walk, The University Series in Higher Math., Van Nostrand, Princeton, N. J., 1964. MR 30 \#1521.

9. C. J. Stone, A local limit theorem for nonlattice multi-dimensional distribution functions, Ann. Math. Statist. 36 (1965), 546-551. MR 30 \#5351.

10. J. L. Teugels, Renewal theorems when the first or the second moment is infinite, Ann. Math. Statist. 39 (1968), 1210-1219. MR 37 \#5952.

11. J. A. Williamson, Random walks and Riesz kernels, Pacific J. Math. 25 (1968), 393-415. MR 37 \#2328.

UNIVERSITY OF WISCONSIN, MADISON, Wisconsin 53706 